\title{
Synthesis of $\beta$-substituted alanines via Michael addition of nucleophiles to dehydroalanine derivatives
}

\author{
Paula M. T. Ferreira, Hernâni L. S. Maia,* Luís S. Monteiro, Joana Sacramento and \\ Joana Sebastião
}

Department of Chemistry, University of Minho, Gualtar, P-4700-320 Braga, Portugal

Received (in Cambridge, UK) 26th April 2000, Accepted 10th July 2000

First published as an Advance Article on the web 13th September 2000

Several $\beta$-substituted alanines are synthesised in high yields by a Michael addition of nucleophiles to $N$-acyl- $N$-(tertbutoxycarbonyl)dehydroalanine methyl ester, using mild reaction conditions and simple work-up procedures. The same method can be applied to dipeptides containing dehydroalanine.

\section{Introduction}

In recent years a large number of non-proteinogenic amino acids have been isolated from plant and bacterial sources. Among these are a group of $\beta$-substituted alanines, some of which exhibit important biological activities, such as $\beta$-(pyrazol-1-yl)alanine which has hypoglycaemic properties, ${ }^{1}$ and quisqualic acid, which possesses potent neuroexcitatory activities. ${ }^{2,3}$ These compounds are isolated from pressed juice of watermelon (Citrullus vulgaris) and from Rangoon Creeper (Quisqualis indica), respectively. ${ }^{4}$ Another heterocyclic $\beta$-substituted alanine, viz. $\beta-(1,2,4$-triazol-1-yl)alanine, is known as an important metabolite (in plants) of the fungicide myclobutanil. ${ }^{5}$

As far back as 1965 , Zahn reported a $96.5 \%$ yield in a synthesis of a $\beta$-substituted alanine by the addition of $N^{\alpha}$-acetylL-lysine to $N$-acetyldehydroalanine ethyl ester in the presence of sodium hydroxide. ${ }^{6}$ Later, a $64 \%$ yield in a Michael addition of benzylamine to Boc- $\Delta \mathrm{Ala}-\mathrm{OMe} \uparrow$ in the absence of inorganic base was obtained. ${ }^{7}$ Recently, other Michael additions of nitrogen nucleophiles to dehydroalanine derivatives have been reported, either in $\mathrm{FeCl}_{3}$-catalysed reactions to give various $\beta$-substituted alanine derivatives in yields varying within the range $13-98 \%{ }^{8}$ or in the presence of inorganic base to give two such compounds, viz. $\beta$-(pyrazol-1-yl)alanine and $\beta-(1,2,4-$ triazol-1-yl)alanine, in fair yields ( 54 and $78 \%$, respectively). ${ }^{9}$ In the latter case, a solid-phase strategy was used to circumvent difficulties met in attempted solution syntheses, namely difficult purification of the products due to similarity of their solubility to that of the corresponding starting materials. Thus, $N$-acetyldehydroalanine was anchored to a Wang resin and treated with nucleophiles in the presence of potassium carbonate under forcing conditions ( 6 to 15 equiv. of nucleophile were used in 2-day reactions at temperatures within the range $\left.50-60{ }^{\circ} \mathrm{C}\right)$.

Our recently reported high-yield synthesis of $N$-acyl- $N$-(tertbutoxycarbonyl)dehydroalanine $\left(\Delta\right.$ Ala) derivatives ${ }^{10,11}$ made these compounds available in large amounts, ready for further applications. Indeed we were able to use these compounds with success as substrates for the Michael reaction, which seemed to be assisted by the second substituent on the nitrogen atom. ${ }^{12} \mathrm{We}$ now report the results obtained in the synthesis of amino acid and peptide derivatives of several $\beta$-substituted alanines by this procedure.

$\dagger \Delta$ Ala means dehydroalanine.

\section{Results and discussion}

The methyl ester of $N, N$-bis(tert-butoxycarbonyl)dehydroalanine [Boc- $\Delta \mathrm{Ala}(N \text {-Boc)-OMe } 1]^{11}$ was treated at room temperature with one mole equivalent of a nucleophile (pyrazole, 1,2,4-triazole or imidazole) in acetonitrile with six mole equivalents of potassium carbonate to give compounds $\mathbf{1 a}, \mathbf{1 b}$ and 1c in excellent yields (Scheme 1 and Table 1 entries 1, 2 and 3, respectively).

Comparison of entries 1 and 2 in Table 1 with the corresponding results of Barbaste et al. ${ }^{9}$, shows that the presence of two substituents on the nitrogen atom greatly increases the reactivity of the $\beta$-carbon atom of $\Delta$ Ala towards nucleophilic attack. This was confirmed when Boc- $\Delta$ Ala-OMe was substituted for $\mathrm{Boc}-\Delta \mathrm{Ala}(N$-Boc)-OMe, as in this case no reaction with pyrazole was detected under the same conditions. This enhanced reactivity allows the reactions to proceed to completion without the need for an excess of nucleophile, thus greatly simplifying the work-up procedures.

The scope of this reaction was further investigated with respect to other nitrogen nucleophiles such as pyrrole, indole and carbazole, but much lower yields were obtained, which may be related to a lower acidity of these compounds as compared with the previous ones. In fact, while no product had been isolated in the attempted reaction with pyrrole, the reaction with 2-formylpyrrole was quantitative and a yield of $82 \%$ was obtained with 2-acetylpyrrole, even when only one mole equivalent of nucleophile was used (Table 1, entries 4-6, compounds 1d-1f, respectively). In the case of indole, the NMR spectrum of the reaction mixture of a 3-day reaction with 1 equiv. of nucleophile showed a 1:1 ratio of addition product to unchanged dehydroamino acid. However, this ratio could be improved to 5:1 when an excess of indole (3 equiv.) was used under identical conditions, and work-up of the reaction mixture gave a $49 \%$ yield of pure $1 \mathrm{~g}$ after recrystallisation (Table 1 , entry 7). Much improved results were also obtained when these heterocycles were activated with an electron-withdrawing substituent (Table 1 entries 8,10-12 and 14-16), but not when an electron-releasing group was placed at the same position (Table 1 entry 9). A broad correlation could be observed between reaction yields and the chemical shift of the nitrogen protons of the heterocyclic nucleophiles; in fact, from the data in Table 1 one may infer that whenever the chemical shift of the nucleophile $\mathrm{NH}$ proton used is equal to or higher than $11.68 \mathrm{ppm}$, as measured in DMSO, no more than 1 equiv. of nucleophile is required for a high yielding reaction. 
Table 1 Results obtained in the synthesis of heterocyclic $\beta$-substituted alanine derivatives by addition of a nucleophile to Boc- $\Delta$ Ala $(N$-Boc)-OMe 1

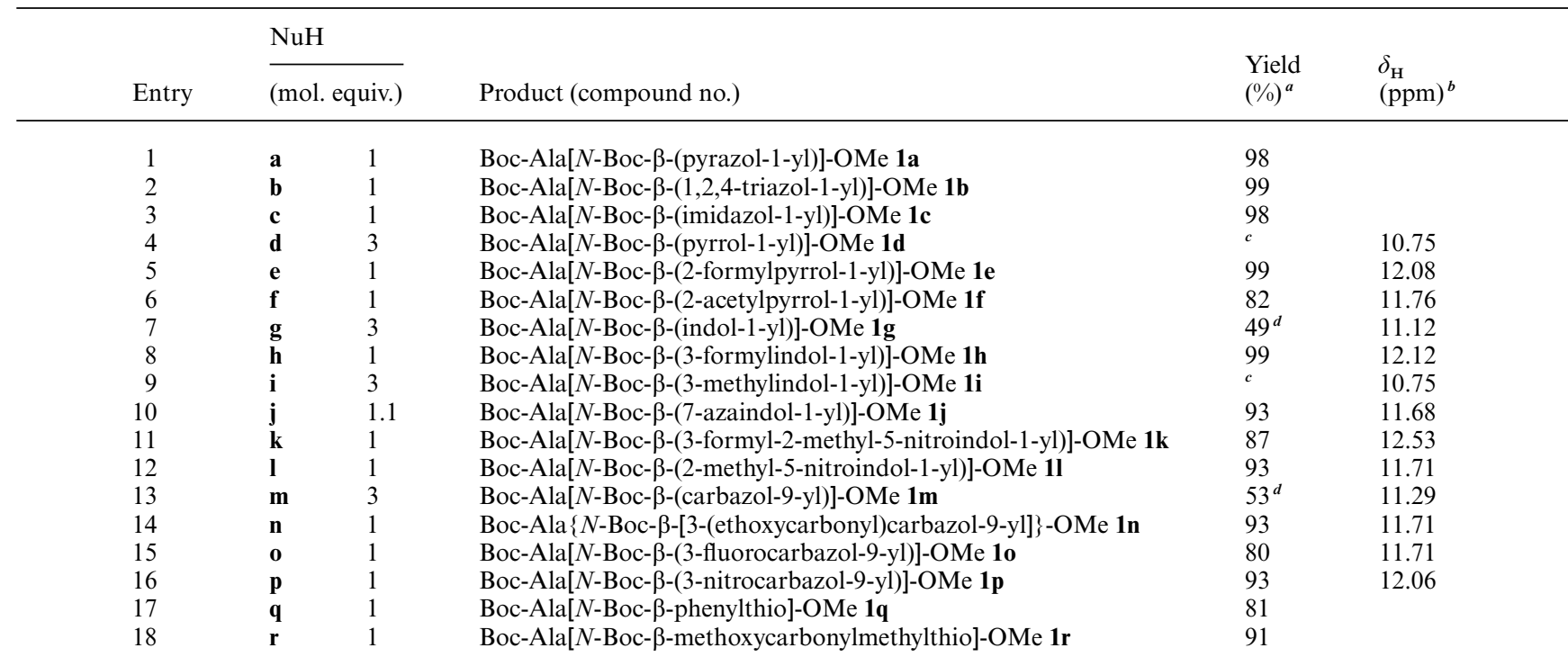

${ }^{a}$ Pure non-recrystallised material. ${ }^{b}$ For the $\mathrm{NH}$ proton of $\mathrm{NuH}\left(300 \mathrm{MHz}\right.$; DMSO; $\left.\mathrm{Me}_{4} \mathrm{Si}\right) .{ }^{c}$ No reaction was detected. ${ }^{d}$ Pure recrystallised material.

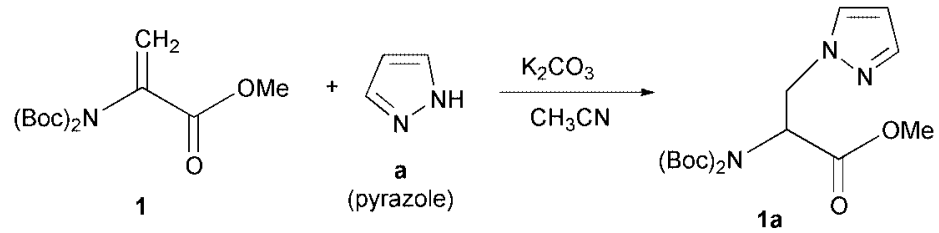<smiles>C=C(NCCCC)C(=O)OCC(C)C(=O)NC(CN)C(=O)OC</smiles>

1

1b-1p

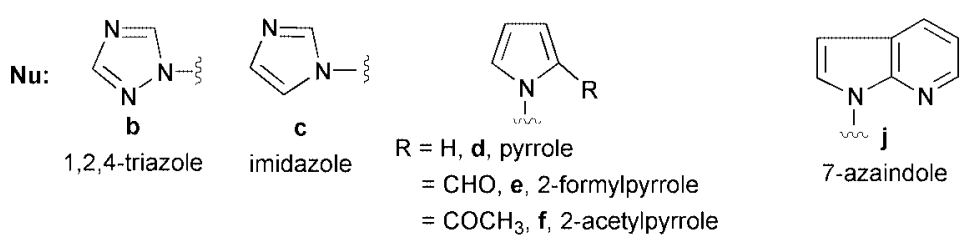<smiles>[R]c1ccc2c(c1)c([R])c([R])n2C</smiles>

$R^{1}, R^{2}, R^{3}=H, g$ indole

$R^{2}=\mathrm{CHO}, \mathrm{R}^{1}, \mathrm{R}^{3}=\mathrm{H}, \mathrm{h}$, 3-formylindole

$\mathrm{R}^{2}=\mathrm{CH}_{3}, \mathrm{R}^{1}, \mathrm{R}^{3}=\mathrm{H}, \mathbf{i}, 3$-methylindole

$\mathrm{R}^{1}=\mathrm{CH}_{3}, \mathrm{R}^{2}=\mathrm{CHO}, \mathrm{R}^{3}=\mathrm{NO}_{2}, \mathbf{k}$,

3-formyl-2-methyl-5-nitroindole

$\mathrm{R}^{1}=\mathrm{CH}_{3}, \mathrm{R}^{2}=\mathrm{H}, \mathrm{R}^{3}=\mathrm{NO}_{2}, \mathrm{l}$,

2-methyl-5-nitroindole<smiles>[R]c1ccc(N(C)c2ccccc2)c(C)c1</smiles>

$\mathrm{R}=\mathrm{H}, \mathbf{m}$, carbazole

$\mathrm{R}=\mathrm{CO}_{2} \mathrm{Et}, \mathrm{n}$, 3-(ethoxycarbonyl)carbazole

$\mathrm{R}=\mathrm{F}, \mathrm{o}, 3$-fluorocarbazole

$\mathrm{R}=\mathrm{NO}_{2}, \mathbf{p}, 3$-nitrocarbazole

Scheme 1

The products $\mathbf{1 c}, \mathbf{1 g}$ and $\mathbf{1 j}$ of the reactions with imidazole, indole and 7-azaindole are isosteres of the corresponding histidine, tryptophan and 7-azatryptophan derivatives, respectively; 7-azatryptophan is used as a fluorescent probe in peptide labelling. ${ }^{9}$

This method was extended successfully to the addition of sulfur nucleophiles, namely thiophenol and methyl mercaptoacetate (Scheme 2, Table 1, entries 17 and 18).

This reaction was also investigated with several dehydroalanine derivatives having unsymmetrical double substitution at their nitrogen atom (Scheme 3, Table 2). In many cases a large amount of Boc- $\Delta$ Ala-OMe was detected in the reaction mixture. We believe that this might have resulted from competitive nucleophilic cleavage of the substituents at the nitrogen atom of the dehydroalanine derivative in a manner similar to that described by Ragnarsson and co-workers ${ }^{13}$ for cleavage of the acyl group from $N$-acyl- $N$-Boc-amines with nitrogen nucleophiles such as tetramethylguanidine and 2-(diethylamino)ethylamine. This competition seems to occur in connection with the 4-nitrobenzoyl group (Table 2, entries 4-6), which can be easily cleaved by nucleophiles, and also with weak nucleophiles such as pyrazole (Table 2, entries 1, 4, 7 and 10). In fact, in the 
Table 2 Results obtained in the synthesis of heterocyclic $\beta$-substituted alanine derivatives by addition to $\mathrm{P}-\Delta \mathrm{Ala}(N$-Boc)-OMe (2-5)

\begin{tabular}{|c|c|c|c|c|}
\hline Entry & $\mathrm{P}$ & $\mathrm{NuH}$ & Product (compound no.) & Yield $(\%)^{a}$ \\
\hline 1 & 2 & $\mathbf{a}$ & Bz-Ala[ $N$-Boc- $\beta$-(pyrazol-1-yl)]-OMe 2a & $53^{b}$ \\
\hline 2 & 2 & b & Bz-Ala $[N$-Boc- $\beta$-(1,2,4-triazol-1-yl)]-OMe $\mathbf{2 b}$ & 98 \\
\hline 3 & 2 & $\mathbf{j}$ & Bz-Ala[ $N$-Boc- $\beta$-(7-azaindol-1-yl)]-OMe $2 \mathbf{j}$ & 92 \\
\hline 4 & 3 & $\mathbf{a}$ & $\mathrm{Bz}\left(\mathrm{NO}_{2}\right)$-Ala[ $N$-Boc- $\beta$-(pyrazol-1-yl) $]-\mathrm{OMe}^{c} \mathbf{3 a}$ & $d$ \\
\hline 5 & 3 & $\mathbf{b}$ & $\mathrm{Bz}\left(\mathrm{NO}_{2}\right)-\mathrm{Ala}[N$-Boc- $\beta-(1,2,4$-triazol-1-yl $)]-\mathrm{OMe} \mathbf{3 b}$ & $67^{e}$ \\
\hline 6 & 3 & $\mathbf{j}$ & $\mathrm{Bz}\left(\mathrm{NO}_{2}\right)-\mathrm{Ala}[N$-Boc- $\beta$-(7-azaindol-1-yl)]-OMe $\mathbf{3 j}$ & $54^{f}$ \\
\hline 7 & 4 & $\mathbf{a}$ & Z-Ala $[N$-Boc- $\beta$-(pyrazol-1-yl)]-OMe 4a & $48^{g}$ \\
\hline 8 & 4 & $\mathbf{b}$ & Z-Ala[ $N$-Boc- $\beta$-(1,2,4-triazol-1-yl)]-OMe $\mathbf{4 b}$ & 98 \\
\hline 9 & 4 & $\mathbf{j}$ & Z-Ala[ $N$-Boc- $\beta$-(7-azaindol-1-yl)]-OMe $4 \mathbf{j}$ & 99 \\
\hline 10 & 5 & $\mathbf{a}$ & $\mathrm{Z}\left(\mathrm{NO}_{2}\right)-\mathrm{Ala}\left[N\right.$-Boc- $\beta$-(pyrazol-1-yl)]-OMe $\mathbf{5} \mathbf{a}^{c}$ & $37^{h}$ \\
\hline 11 & 5 & b & $\mathrm{Z}\left(\mathrm{NO}_{2}\right)-\mathrm{Ala}[N$-Boc- $\beta$-(1,2,4-triazol-1-yl)]-OMe 5b & 98 \\
\hline 12 & 5 & $\mathbf{g}$ & $\mathrm{Z}\left(\mathrm{NO}_{2}\right)-\mathrm{Ala}[N$-Boc- $\beta$-(indol-1-yl)]-OMe 5g & 46 \\
\hline 13 & 5 & $\mathbf{j}$ & $\mathrm{Z}\left(\mathrm{NO}_{2}\right)$-Ala $[N$-Boc- $\beta$-(7-azaindol-1-yl) $]$-OMe $5 \mathbf{j}$ & 87 \\
\hline
\end{tabular}

${ }^{a}$ Pure non-recrystallised material. ${ }^{b}$ Boc- $\Delta$ Ala-OMe was also isolated, in $22 \%$ yield. ${ }^{c} \mathrm{Bz}\left(\mathrm{NO}_{2}\right)=4$-nitrobenzoyl, $\mathrm{Z}\left(\mathrm{NO}_{2}\right)=4$-nitrobenzyloxycarbonyl. ${ }^{d}$ Only Boc- $\Delta$ Ala-OMe was isolated, in $82 \%$ yield. ${ }^{e}$ Boc- $\Delta$ Ala-OMe was also isolated, in $28 \%$ yield. ${ }^{f}$ Boc- $\Delta$ Ala-OMe was also isolated, in $42 \%$ yield. ${ }^{g}$ Boc- $\Delta$ Ala-OMe was also isolated, in $30 \%$ yield. ${ }^{h}$ Boc- $\Delta$ Ala-OMe was also isolated, in $45 \%$ yield.

Table 3 Yields in the selective cleavage of heterocyclic $\beta$-substituted alanine derivatives

\begin{tabular}{|c|c|c|c|}
\hline Reactant & $\begin{array}{l}\text { Deprotection } \\
\text { reactant }\end{array}$ & Product & Yield $(\%)$ \\
\hline $\mathbf{1 b}$ & TFA & H-Ala[ $\beta$-(1,2,4-triazol-1-yl)]-OMe·2TFA $\mathbf{6 b}$ & 80 \\
\hline $\mathbf{1 j}$ & TFA & H-Ala[ $\beta$-(7-azaindol-1-yl)]-OMe·TFA $\mathbf{6 j}$ & 85 \\
\hline $1 \mathrm{~m}$ & TFA & H-Ala $[\beta$-(carbazol-9-yl)]-OMe·TFA 6m & 91 \\
\hline $\mathbf{2 b}$ & DEAEA & Boc-Ala[ $\beta$-(1,2,4-triazol-1-yl)]-OMe $7 \mathbf{b}$ & 78 \\
\hline $\mathbf{5 b}$ & $\mathrm{Al} / \mathrm{Hg}$ & Boc-Ala[ $\beta$-(1,2,4-triazol-1-yl)]-OMe 7b & 86 \\
\hline $1 \mathbf{a}$ & $\mathrm{NaOH}$ & Boc-Ala $[N$-Boc- $\beta$-(pyrazol-1-yl) $]-O H ~ 8 a$ & 91 \\
\hline $1 \mathrm{~b}$ & $\mathrm{NaOH}$ & Boc-Ala $[N$-Boc- $\beta$-( $1,2,4$-triazol-1-yl)]-OH $8 \mathbf{b}$ & 86 \\
\hline $1 \mathrm{~h}$ & $\mathrm{NaOH}$ & Boc-Ala[ $N$-Boc- $\beta$-(3-formylindol-1-yl)]-OH $\mathbf{8 h}$ & 89 \\
\hline $\mathbf{1 j}$ & $\mathrm{NaOH}$ & Boc-Ala $[N$-Boc- $\beta$-(7-azaindol-1-yl)]-OH 8j & 94 \\
\hline
\end{tabular}

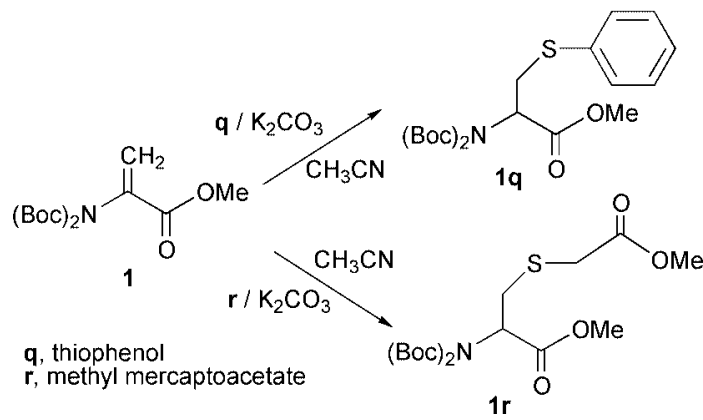

Scheme 2

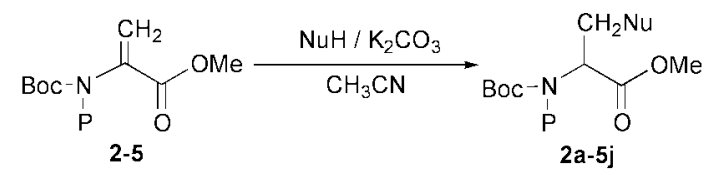

$$
\begin{aligned}
& \text { P: benzoyl, (Bz), } 2 \\
& \text { 4-nitrobenzoyl, }\left[\mathrm{Bz}\left(\mathrm{NO}_{2}\right)\right], 3 \\
& \text { benzyloxycarbonyl, ( } \mathrm{Z} \text { ) } 4 \\
& \text { 4-nitrobenzyloxycarbonyl, [Z( } \left.\left(\mathrm{NO}_{2}\right)\right], 5
\end{aligned}
$$

Scheme 3

case of the strong nucleophile 1,2,4-triazole all reactions were almost quantitative except for that with the 4-nitrobenzoyl derivative (Table 2, entries 2, 5,8 and 11), thus showing that competitive cleavage may be overcome when the nucleophile is sufficiently strong to provide fast addition. Slightly lower yields were obtained in the corresponding reactions with the weaker nucleophile 7-azaindole (Table 2, entries 3, 6, 9 and 13).

As shown in Table 3, the Boc groups can be easily removed from the $N, N$-bis(tert-butoxycarbonyl) $\beta$-substituted alanine methyl esters by treatment with TFA, as exemplified in the preparation of compounds $6 \mathbf{b}, \mathbf{6 j}$ and $\mathbf{6 m}$. Although in most cases the use of a combination of two different substituents at the $\alpha$-amine nitrogen atom does not improve the yields of addition product, it allows selective cleavage of one of these substituents to yield an N-protected product. Thus, 2-(diethylamino)ethylamine (DEAEA $)^{13}$ was used to cleave benzoyl from $\mathbf{2 b}$, while 4 nitrobenzyloxycarbonyl was cleaved from $\mathbf{5 b}$ by reduction with mercury-activated aluminium. ${ }^{14}$ In both cases Boc-Ala[ $\beta-(1,2,4-$ triazol-1-yl)]-OMe $7 \mathbf{b}$ was obtained in good yields as shown in Table 3. Saponification of the $N, N$-bis(tert-butoxycarbonyl) amino acid methyl esters gives the corresponding $N, N$-bis(tertbutoxycarbonyl) amino acids, as exemplified in the preparation of compounds $\mathbf{8 a}, \mathbf{8 b}, \mathbf{8 h}$ and $\mathbf{8 j}$ (Table 3). Any of these Nprotected compounds can be readily used for peptide-chain elongation. Thus, by using standard dicyclohexylcarbodiimide (DCC) couplings, compounds 8a and $\mathbf{8 j}$ (Table 3) were treated with H-L-Phe-OEt to yield Boc-DL-Ala[ $N$-Boc- $\beta$-(pyrazol-1yl)]-L-Phe-OEt 9a and Boc-DL-Ala[ $N$-Boc- $\beta$-(7-azaindol-1-yl)]L-Phe-OEt $9 \mathbf{j}$ in yields of 80 and $86 \%$, respectively. HPLC analysis of the reaction products indicated 1:1 mixtures of diastereomers, which shows that, as expected, the addition reaction gives rise to a racemic mixture with regard to the chiral centre generated within the $\beta$-substituted alanine residue.

The method described above can also be applied for direct reaction of nitrogen nucleophiles with dipeptides containing dehydroalanine. Table 4 shows the results obtained in the addition of various heterocyclic nucleophiles to either Tos$\operatorname{Gly}(N$-Boc)- $\Delta \mathrm{Ala}(N$-Boc)-OMe or $\operatorname{Boc}-\mathrm{Ala}(N$-Boc $)-\Delta \mathrm{Ala}(N$ Boc)-OMe.

Our results show that $N, N$-disubstituted dehydroamino acid residues are good substrates for nucleophilic attack in a Michael reaction, which allows the synthesis of a variety of $\beta$ substituted alanine derivatives under mild conditions and using very simple work-up procedures; in most cases the products are obtained in quantitative yields. Boc- $\Delta \mathrm{Ala}(N$-Boc)-OMe proved to be an excellent substrate for nucleophilic attack, although other combinations of $\mathrm{N}$-acyl groups can also be used, which increases the versatility of the method. 
Table 4 Results obtained in the synthesis of heterocyclic $\beta$-substituted alanine peptides by addition of a nucleophile to Tos-Gly $(N$-Boc)- $\Delta$ Ala $(N$ Boc)-OMe or Boc-Ala $(N$-Boc $)-\Delta$ Ala $(N$-Boc $)-O M e$

\begin{tabular}{|c|c|c|c|}
\hline Entry & $\mathrm{NuH}$ & Product (compound no.) & Yield $(\%)$ \\
\hline 1 & $\mathbf{a}$ & $\operatorname{Tos}-\mathrm{Gly}(N$-Boc)-Ala[$N$-Boc- $\beta$-(pyrazol-1-yl)]-OMe 10a & 76 \\
\hline 2 & b & $\operatorname{Tos}-\mathrm{Gly}(N$-Boc)-Ala[$[N$-Boc- $\beta-(1,2,4$-triazol-1-yl) $]-O M e \mathbf{1 0 b}$ & 99 \\
\hline 3 & c & $\operatorname{Tos}-\mathrm{Gly}(N$-Boc)-Ala[ $N$-Boc- $\beta$-(imidazol-1-yl)]-OMe 10c & 75 \\
\hline 4 & $\mathbf{h}$ & Tos-Gly $(N$-Boc)-Ala[ $N$-Boc- $\beta$-(3-formylindol-1-yl)]- OMe 10h & 74 \\
\hline 5 & $\mathbf{j}$ & Tos-Gly $(N$-Boc)-Ala[ $N$-Boc- $\beta$-(7-azaindol-1-yl)]-OMe 10j & 90 \\
\hline 6 & b & $\operatorname{Boc}-\mathrm{Ala}(N$-Boc)-Ala[$N$-Boc- $\beta$-(1,2,4-triazol-1-yl)]-OMe 11b & 98 \\
\hline
\end{tabular}

\section{Experimental}

\section{General methods}

All mps were determined on a Gallenkamp melting point apparatus and are uncorrected. TLC analyses were carried out on $0.25 \mathrm{~mm}$ thick precoated silica plates (Merck Fertigplatten Kieselgel $60 \mathrm{~F}_{254}$ ) and spots were visualised under UV or by exposure to vaporised iodine. Preparative chromatography was carried out on Merck Kieselgel 60 (230-400 mesh). ${ }^{1} \mathrm{H}$ NMR spectra were recorded on a Varian 300 spectrometer for samples in $\approx 5 \% \mathrm{CDCl}_{3}$ solution at $25^{\circ} \mathrm{C}$, when not otherwise stated. All shifts are given in $\delta$ ppm using $\delta_{\mathrm{H}} \mathrm{Me}_{4} \mathrm{Si}=0$ as reference. $J$-Values are given in Hz. Assignments were made by comparison of chemical shifts, peak multiplicities and $J$-values. ${ }^{13} \mathrm{C}$ NMR spectra were determined in the same instrument at 75.4 $\mathrm{MHz}$ using the solvent peak as internal reference. Elemental analyses of crystalline derivatives and some oils were carried out on a Leco CHNS 932 instrument. Analysis of diastereomers by HPLC experiments was run on a Shimadzu instrument, type $6 \mathrm{~A}$, connected to a Merck prepacked column, type Hibar RT 250-4, filled with LiChrospher $100 \mathrm{CH}-18 / 2(5 \mu \mathrm{m})$ and the eluent was a mixture of acetonitrile and water. The peaks were measured with a Shimadzu integrator, type C-R6A Chromatopack.

\section{Preparation of dehydroalanine derivatives}

Synthesis of Boc- $\Delta \mathrm{Ala}(N$-Boc)-OMe $1, \operatorname{Bz}-\Delta \mathrm{Ala}(N$-Boc)OMe 2, Z- $\Delta \mathrm{Ala}\left(N\right.$-Boc)-OMe 4 and $\mathrm{Z}\left(\mathrm{NO}_{2}\right)-\Delta \mathrm{Ala}(N$-Boc)OMe 5. These compounds have been described elsewhere. ${ }^{11}$

Synthesis of $\mathrm{Bz}\left(\mathrm{NO}_{2}\right)-\Delta \mathrm{Ala}(\mathrm{N}$-Boc)-OMe 3. The same procedure as above was used in a 5-mmolar scale to synthesise this compound in $71 \%$ yield, mp $104-104.5^{\circ} \mathrm{C}$ (from ethyl acetatediethyl ether) (Found: $\mathrm{C}, 54.9 ; \mathrm{H}, 5.2 ; \mathrm{N}, 7.95$. Calc. for $\left.\mathrm{C}_{16} \mathrm{H}_{18} \mathrm{~N}_{2} \mathrm{O}_{7}: \mathrm{C}, 54.9 ; \mathrm{H}, 5.2 ; \mathrm{N}, 8.0 \%\right) ; \delta_{\mathrm{H}} 1.29(9 \mathrm{H}, \mathrm{s}, \mathrm{Boc})$, $3.86(3 \mathrm{H}, \mathrm{s}, \mathrm{OMe}), 5.91\left(1 \mathrm{H}, \mathrm{s}, \beta \mathrm{CH}_{2}\right), 6.60\left(1 \mathrm{H}, \mathrm{s}, \beta \mathrm{CH}_{2}\right), 7.85$ [ $\left.2 \mathrm{H}, \mathrm{d}, J 8.7, \mathrm{Bz}\left(\mathrm{NO}_{2}\right)\right], 8.31\left[2 \mathrm{H}, \mathrm{d}, J 8.7, \mathrm{Bz}\left(\mathrm{NO}_{2}\right)\right] ; \delta_{\mathrm{C}} 27.50$, $52.85,84.90,98.55,123.46,126.91,128.65,135.10,142.07$, $149.22,150.94,163.41$.

Synthesis of Boc-Ala $(N$-Boc)- $\Delta \operatorname{Ala}(N$-Boc)-OMe 11. This compound has been described previously. ${ }^{11}$

Synthesis of Tos-Gly $(N$-Boc $)-\Delta \operatorname{Ala}(N$-Boc $)-O M e ~ 10$. The same procedure as above was used in a 5 -mmolar scale to synthesise this compound in $96 \%$ yield, mp $60.5-62.5^{\circ} \mathrm{C}$ (from diethyl ether- $n$-hexane) (Found: C, $54.2 ; \mathrm{H}, 6.3 ; \mathrm{N}, 5.3 ; \mathrm{S}, 6.3$. Calc. for $\mathrm{C}_{23} \mathrm{H}_{32} \mathrm{~N}_{2} \mathrm{O}_{9} \mathrm{~S}$ : C, 53.9; H, 6.3; N, 5.5; S, 6.2\%); $\delta_{\mathrm{H}} 1.24$ (9 H, s, Boc), 1.49 (9 H, s, Boc), 2.43 (3 H, s, $\mathrm{CH}_{3}$ Tos), 3.80 (3 H, s, OMe), $5.22\left(2 \mathrm{H}, \mathrm{s}, \mathrm{CH}_{2} \mathrm{Gly}\right), 5.74\left(1 \mathrm{H}, \mathrm{d}, \mathrm{J} 0.6, \beta \mathrm{CH}_{2}\right.$ $\Delta$ Ala), 6.52 (1 H, d, $J 0.6, \beta \mathrm{CH}_{2} \Delta$ Ala $), 7.29(2 \mathrm{H}, \mathrm{d}, J 8.4, \mathrm{ArH}$ T), $7.93(2 \mathrm{H}, \mathrm{d}, J 8.4, \mathrm{ArH} \mathrm{T}) ; \delta_{\mathrm{C}} 21.62,27.76,27.79,51.10$, $52.48,84.58,84.63,126.78,128.72,129.00,134.47,136.77$, $144.17,150.52,151.19,163.29,170.36$

\section{Michael addition to $\mathrm{N}, \mathrm{N}$-bis(tert-butoxycarbonyl)dehydro- alanine methyl ester}

General method. As described elsewhere, ${ }^{12}$ to a solution of
$1 \mathrm{mmol}$ of Boc- $\Delta \mathrm{Ala}\left(N\right.$-Boc)-OMe in acetonitrile $\left(10 \mathrm{~cm}^{3}\right)$ was added $\mathrm{K}_{2} \mathrm{CO}_{3}$ (6 equiv.), followed by the nucleophile (1 equiv.) with rapid stirring at room temperature. The reaction was monitored by TLC and when no starting material was detected, the solution was filtered and evaporated at reduced pressure to give the required product.

Synthesis of $1 \mathrm{a}, \mathbf{1 b}, \mathbf{1 c}, \mathbf{1 g}, \mathbf{1 h}, \mathbf{1 j}$ and $1 \mathrm{n}$. These compounds have been synthesised as described elsewhere. ${ }^{12}$

Synthesis of 1e. The above general method was used with 2-formylpyrrole to prepare this compound as a crystalline material $(99 \%)$, mp $73-74{ }^{\circ} \mathrm{C}$ (from diethyl ether- $n$-hexane) (Found: C, 57.6; H, 7.1; N, 7.0. Calc. for $\mathrm{C}_{19} \mathrm{H}_{28} \mathrm{~N}_{2} \mathrm{O}_{7}$ : C, 57.6; $\mathrm{H}, 7.1 ; \mathrm{N}, 7.1 \%) ; \delta_{\mathrm{H}} 1.41(18 \mathrm{H}, \mathrm{s}, \mathrm{Boc}), 3.78(3 \mathrm{H}, \mathrm{s}, \mathrm{OMe}), 4.54$ $\left(1 \mathrm{H}, \mathrm{dd}, J 10.2\right.$ and $\left.13.8, \beta \mathrm{CH}_{2}\right), 5.25(1 \mathrm{H}, \mathrm{dd}, J 4.5$ and 13.8 , $\left.\beta \mathrm{CH}_{2}\right), 5.48(1 \mathrm{H}, \mathrm{dd}, J 4.5$ and $10.2, \alpha \mathrm{CH}), 6.21(1 \mathrm{H}, \mathrm{dd}, J 2.7$ and 4.1, 4-H pyr.), 6.81 ( $1 \mathrm{H}, \mathrm{m}, 3-\mathrm{H}$ pyr.), 6.93 (1 H, dd, $J 1.5$ and $4.1,5-\mathrm{H}$ pyr.), $9.53(1 \mathrm{H}, \mathrm{s}, \mathrm{CHO}) ; \delta_{\mathrm{C}} 27.82,48.45,52.35$, $57.95,83.34,109.80,120.86,131.64,132.22,151.44,169.03$, 179.30 .

Synthesis of 1f. The above general method was used with 2-acetylpyrrole to prepare this compound as an oil which failed to crystallise $(82 \%) ; \delta_{\mathrm{H}} 1.40(18 \mathrm{H}, \mathrm{s}, \mathrm{Boc}), 2.42\left(3 \mathrm{H}, \mathrm{s}, \mathrm{CH}_{3} \mathrm{CO}\right)$, $3.78(3 \mathrm{H}, \mathrm{s}, \mathrm{OMe}), 4.47\left(1 \mathrm{H}, \mathrm{dd}, J 10.2\right.$ and $\left.12.0, \beta \mathrm{CH}_{2}\right), 5.29$ $\left(1 \mathrm{H}, \mathrm{dd}, J 13.8\right.$ and $\left.9.0, \beta_{C_{2}}\right), 5.54(1 \mathrm{H}, \mathrm{dd}, J 10.2$ and 7.4 , $\alpha \mathrm{CH}), 6.12(1 \mathrm{H}, \mathrm{dd}, J 3.9$ and 3.2, 4-H pyr.), $6.71(1 \mathrm{H}, \mathrm{m}, 3-\mathrm{H}$ pyr.), $6.94\left(1 \mathrm{H}\right.$, dd, $J 4.2$ and $3,5-\mathrm{H}$, pyr.); $\delta_{\mathrm{C}} 26.99,27.83$, $49.15,52.30,58.10,83.14,108.33,120.38,130.61,131.18$, $151.53,169.25,188.31$.

Synthesis of $1 \mathbf{k}$. The above general method was used with 3-formyl-2-methyl-5-nitroindole to prepare this compound as a crystalline material $(87 \%)$, mp $161-162^{\circ} \mathrm{C}$ (from diethyl ether$n$-hexane) (Found: C, 56.9; $\mathrm{H}, 6.1 ; \mathrm{N}, 8.3$. Calc. for $\mathrm{C}_{24} \mathrm{H}_{31} \mathrm{~N}_{3} \mathrm{O}_{9}$ : C, 57.0; H, 6.2; N, 8.3\%); $\delta_{\mathrm{H}} 1.28(18 \mathrm{H}, \mathrm{s}, \mathrm{Boc}), 2.78(3 \mathrm{H}, \mathrm{s}$, 2- $\mathrm{CH}_{3}$ ind.), 3.83 (3 H, s, OMe), 4.81-4.98 (2 H, complex signal, $\left.\beta \mathrm{CH}_{2}\right), 5.36(1 \mathrm{H}, \mathrm{dd}, J 4.5$ and $10.2, \alpha \mathrm{CH}), 7.42(1 \mathrm{H}, \mathrm{d}, J 9.3$, 7-H ind.), 8.16 ( $1 \mathrm{H}, \mathrm{dd}, J 2.1$ and 9.3, 6- $\mathrm{H}$ ind.), 9.17 ( $1 \mathrm{H}, \mathrm{d}$, $J$ 2.1, 4-H ind.), 10.22 ( $1 \mathrm{H}, \mathrm{s}, 3-\mathrm{CHO}) ; \delta_{\mathrm{C}} 10.54,27.63,43.57$, $52.77,56.83,84.32,109.65,115.82,117.57,118.71,125.25$, $139.85,143.95,150.56,151.81,168.33,183.86$.

Synthesis of 11. The above general method was used with 2-methyl-5-nitroindole to prepare this compound as a crystalline material $(93 \%), \mathrm{mp} 110-111^{\circ} \mathrm{C}$ (from diethyl ether- $n$ hexane) (Found: C, 57.7; H, 6.5; N, 8.8. Calc. for $\mathrm{C}_{23} \mathrm{H}_{31} \mathrm{~N}_{3} \mathrm{O}_{8}$ : $\mathrm{C}, 57.85 ; \mathrm{H}, 6.5 ; \mathrm{N}, 8.8 \%) ; \delta_{\mathrm{H}} 1.28(18 \mathrm{H}, \mathrm{s}, \mathrm{Boc}), 2.46(3 \mathrm{H}, \mathrm{s}, 2-$ $\mathrm{CH}_{3}$ ind.), 3.82 (3 H, s, OMe), 4.77 (12 H, dd, $J 4.5$ and 15.3, $\left.\beta \mathrm{CH}_{2}\right), 4.87\left(1 \mathrm{H}, \mathrm{dd}, J 9.9\right.$ and $\left.15.3, \beta \mathrm{CH}_{2}\right), 5.29(1 \mathrm{H}, \mathrm{dd}, J 4.5$ and 9.9, $\alpha \mathrm{CH}), 6.43(1 \mathrm{H}, \mathrm{s}, 3-\mathrm{H}$ ind.), $7.30(1 \mathrm{H}, \mathrm{d}, J$ 9.0, 7-H ind.), $8.02(1 \mathrm{H}, \mathrm{dd}, J 2.4$ and $9.0,6-\mathrm{H}$ ind.), $8.44(1 \mathrm{H}, \mathrm{d}, J 2.4$, $4-\mathrm{H}$ ind.); $\delta_{\mathrm{C}} 12.61,27.61,43.39,52.56,57.69,83.77,103.17$, $108.76,116.39,116.53,127.48,140.33,141.58,151.68,168.77$.

Synthesis of $1 \mathrm{~m}$. The above general method was used with carbazole to prepare this compound as an oily material, which 
was crystallised from diethyl ether- $n$-hexane to give a pure crystalline product $(53 \%), \mathrm{mp} 120-121^{\circ} \mathrm{C}$ (from diethyl ether- $n$ hexane) (Found: $\mathrm{C}, 66.9 ; \mathrm{H}, 7.0 ; \mathrm{N}, 5.9$. Calc. for $\mathrm{C}_{26} \mathrm{H}_{32} \mathrm{~N}_{2} \mathrm{O}_{6}$ : $\mathrm{C}, 66.65 ; \mathrm{H}, 6.9 ; \mathrm{N}, 6.0 \%) ; \delta_{\mathrm{H}} 1.18(18 \mathrm{H}, \mathrm{s}, \mathrm{Boc}), 3.83(3 \mathrm{H}, \mathrm{s}$, $\mathrm{OMe}), 4.90\left(2 \mathrm{H}\right.$, comp. signal, $\left.\beta \mathrm{CH}_{2}\right), 5.46(1 \mathrm{H}, \mathrm{dd}, J 4.2$ and 10.2, $\alpha \mathrm{CH}), 7.22-8.07$ (8 H, complex signal, ArH carb.); $\delta_{\mathrm{C}} 27.45,42.87,52.44,57.40,83.21,108.55,119.21,120.16$, $123.08,125.75,140.41,151.42,169.43$.

Synthesis of 10. The above general method was used with 3-fluorocarbazole to prepare this compound as a crystalline material $(80 \%), \mathrm{mp} 131.5-133{ }^{\circ} \mathrm{C}$ (from diethyl ether- $n$-hexane) (Found: C, 64.0; $\mathrm{H}, 6.6 ; \mathrm{N}, 5.9$. Calc. for $\mathrm{C}_{26} \mathrm{H}_{31} \mathrm{FN}_{2} \mathrm{O}_{6}$ : C, 64.2; $\mathrm{H}, 6.4 ; \mathrm{N}, 5.8 \%) ; \delta_{\mathrm{H}} 1.19(18 \mathrm{H}, \mathrm{s}, \mathrm{Boc}), 3.83$ (3 H, s, OMe), 4.97 $\left(1 \mathrm{H}, \mathrm{dd}, J 4.6\right.$ and $\left.15.4, \beta \mathrm{CH}_{2}\right), 5.06(1 \mathrm{H}, \mathrm{dd}, J 9.2$ and 15.4 , $\left.\beta \mathrm{CH}_{2}\right), 5.42(1 \mathrm{H}, \mathrm{dd}, J 4.6$ and $9.2, \alpha \mathrm{CH}), 7.13-7.48(5 \mathrm{H}$, complex signal, 1-H, 2-H, 6-H, 7-H, 8-H carb.), $7.70(1 \mathrm{H}$, dd, $J 2.4$ and $8.9,5-\mathrm{H}$ carb.), $8.00(1 \mathrm{H}, \mathrm{d}, J$ 7.9, 4-H carb.); $\delta_{\mathrm{C}} 27.52,43.35,52.65,56.96,83.64,108.49,109.61,117.02$, $120.85,121.16,121.62,122.86,122.90,127.57,140.94,141.63$, $143.76,151.62,168.96$.

Synthesis of 1p. The above general method was used with 3-nitrocarbazole to prepare this compound as a crystalline material $(93 \%), \mathrm{mp} 142.5-143{ }^{\circ} \mathrm{C}$ (from diethyl ether- $n$-hexane) (Found: $\mathrm{C}, 60.7 ; \mathrm{H}, 6.1 ; \mathrm{N}, 8.1$. Calc. for $\mathrm{C}_{26} \mathrm{H}_{31} \mathrm{~N}_{3} \mathrm{O}_{8}$ : C, 60.8; $\mathrm{H}, 6.1 ; \mathrm{N}, 8.2 \%) ; \delta_{\mathrm{H}} 1.20(18 \mathrm{H}, \mathrm{s}, \mathrm{Boc}), 3.84(3 \mathrm{H}, \mathrm{s}, \mathrm{OMe}), 5.03$ $\left(1 \mathrm{H}, \mathrm{dd}, J 4.8\right.$ and $\left.15.2, \beta \mathrm{CH}_{2}\right), 5.13(1 \mathrm{H}, \mathrm{dd}, J 9.6$ and 15.2 , $\left.\beta \mathrm{CH}_{2}\right), 5.46(1 \mathrm{H}, \mathrm{dd}, J 4.8$ and 9.6, $\alpha \mathrm{CH}), 7.27-7.59(4 \mathrm{H}$, complex signal, 1-, 6-, 7- and 8-H carb.), 8.13 (1 H, d, $J$ 8.1, 2-H carb.), $8.37(1 \mathrm{H}, \mathrm{dd}, J 2.4$ and 9.0, 5- $\mathrm{H}$ carb. $), 8.99(1 \mathrm{H}, \mathrm{d}$, $J$ 2.1, 4-H carb.); $\delta_{\mathrm{C}} 27.53,43.37,52.68,56.96,83.69,108.50$, $109.61,117.07,120.89,121.19,121.67,122.86,122.93,127.53$, $141.10,142.02,145.05,151.62,169.03$.

Synthesis of 1q. The above general method was used with thiophenol to prepare this compound as a crystalline material (81\%), mp $68-70{ }^{\circ} \mathrm{C}$ (from diethyl ether- $n$-hexane) (Found: $\mathrm{C}$, 58.2; H, 7.1; N, 3.65; S, 7.7. Calc. for $\mathrm{C}_{20} \mathrm{H}_{29} \mathrm{NO}_{6} \mathrm{~S}: \mathrm{C}, 58.4 ; \mathrm{H}$, $7.1 ; \mathrm{N}, 3.4 ; \mathrm{S}, 7.8 \%) ; \delta_{\mathrm{H}} 1.47(18 \mathrm{H}, \mathrm{s}, \mathrm{Boc}), 3.49(1 \mathrm{H}, \mathrm{dd}, J 9.9$ and $\left.14.6, \beta \mathrm{CH}_{2}\right), 3.73(3 \mathrm{H}, \mathrm{s}, \mathrm{OMe}), 3.76(1 \mathrm{H}, \mathrm{dd}, J 4.8$ and 14.6, $\left.\beta \mathrm{CH}_{2}\right), 5.12(1 \mathrm{H}, \mathrm{dd}, J 4.8$ and $9.9, \alpha \mathrm{CH}), 7.20-7.54(5 \mathrm{H}$, $\mathrm{m}, \mathrm{ArH}) ; \delta_{\mathrm{C}} 27.86,34.76,52.38,57.78,83.24,126.40,128.94$, $129.68,135.59,151.76,170.16$.

Synthesis of 1r. The above general method was used with methyl mercaptoacetate to prepare this compound as a crystalline material $(91 \%), \mathrm{mp} 50.5-52{ }^{\circ} \mathrm{C}$ (from diethyl ether- $n$ hexane) (Found: $\mathrm{C}, 50.4 ; \mathrm{H}, 7.3 ; \mathrm{N}, 3.55$. Calc. for $\mathrm{C}_{17} \mathrm{H}_{29} \mathrm{NO}_{8} \mathrm{~S}$ : C, 50.1; H, 7.2; N, 3.4\%); $\delta_{\mathrm{H}} 1.48(18 \mathrm{H}, \mathrm{s}, \mathrm{Boc}), 3.07-3.44(4 \mathrm{H}$, $\mathrm{m}, \beta \mathrm{CH}_{2}+\mathrm{CH}_{2}$ thiol $), 3.71\left(6 \mathrm{H}, \mathrm{s}, \mathrm{CH}_{3}\right.$ thiol $\left.+\mathrm{OMe}\right), 5.11$ $(1 \mathrm{H}, \mathrm{dd}, J 5.4$ and $9.5, \alpha \mathrm{CH}) ; \delta_{\mathrm{C}} 27.04,32.74,33.11,52.31$, $52.36,57.12,83.39,151.86,169.96,170.48$.

\section{Michael addition to $\mathrm{N}$-acyl- $\mathrm{N}$-(tert-butyloxycarbonyl)dehydro- alanine methyl ester}

General method. The method described above for addition of nucleophiles to Boc- $\Delta \mathrm{Ala}(N$-Boc)-OMe was used, yielding in most cases an oil; this was then purified by chromatography through a silica column and using diethyl ether- $n$-hexane as the eluent.

Synthesis of 2a. The above general method was used with $\mathrm{Bz}-\Delta \mathrm{Ala}(N$-Boc)-OMe and pyrazole to prepare this compound as a crystalline material $(53 \%), \mathrm{mp} 50.0-51.5^{\circ} \mathrm{C}$ (from diethyl ether- $n$-hexane) (Found: $\mathrm{C}, 61.0 ; \mathrm{H}, 6.3 ; \mathrm{N}, 11.2$. Calc. for $\left.\mathrm{C}_{19} \mathrm{H}_{23} \mathrm{~N}_{3} \mathrm{O}_{5}: \mathrm{C}, 61.1 ; \mathrm{H}, 6.2 ; \mathrm{N}, 11.25 \%\right) ; \delta_{\mathrm{H}} 1.09(9 \mathrm{H}, \mathrm{s}, \mathrm{Boc})$, $3.81(3 \mathrm{H}, \mathrm{s}, \mathrm{OMe}), 4.94\left(2 \mathrm{H}, \mathrm{m}, \beta \mathrm{CH}_{2}\right), 5.70(1 \mathrm{H}, \mathrm{dd}, J 6.0$ and $8.9, \alpha \mathrm{CH}), 6.23(1 \mathrm{H}, \mathrm{t}, J 2.4,4-\mathrm{H}$ pyr.), 7.34-7.51 $(7 \mathrm{H}, \mathrm{m}$,
$\mathrm{Bz}+3-\mathrm{H}+5-\mathrm{H}$ pyr. $) ; \delta_{\mathrm{C}} 27.19,50.86,52.70,57.93,83.93$, $105.71,127.70,127.88,130.23,131.26,136.90,140.14,152.36$, $168.88,172.81$.

Synthesis of $\mathbf{2 b}$. The above general method was used with $\mathrm{Bz}-\Delta \mathrm{Ala}(N$-Boc)-OMe and 1,2,4-triazole to prepare this compound; in this case the crude product was crystalline and did not require further purification $(98 \%), \mathrm{mp} 84.0-85.5^{\circ} \mathrm{C}$ (from diethyl ether- $n$-hexane) (Found: C, $58.0 ; \mathrm{H}, 6.1 ; \mathrm{N}, 15.2$. Calc. for $\mathrm{C}_{18} \mathrm{H}_{22} \mathrm{~N}_{4} \mathrm{O}_{5}$ : C, 57.75; H, 5.9; N, 15.0\%); $\delta_{\mathrm{H}} 1.08(9 \mathrm{H}, \mathrm{s}$, Boc), $3.82(3 \mathrm{H}, \mathrm{s}, \mathrm{OMe}), 4.98\left(2 \mathrm{H}, \mathrm{d}, J 7.5, \beta \mathrm{CH}_{2}\right), 5.66(1 \mathrm{H}, \mathrm{t}$, $J$ 7.5, $\alpha \mathrm{CH}), 7.40(5 \mathrm{H}, \mathrm{m}, \mathrm{Bz}), 7.94(1 \mathrm{H}, \mathrm{s}, 3-$ or $5-\mathrm{H}$ triaz.), $8.13(1 \mathrm{H}, \mathrm{s}, 5-$ or $3-\mathrm{H}$ triaz. $) ; \delta_{\mathrm{C}} 27.10,48.49,52.90,57.14$, $84.43,127.58,128.07,131.57,136.54,143.18,144.19,152.41$, $168.40,172.79$

Synthesis of $\mathbf{2 j}$. The above general method was used with $\mathrm{Bz}-\Delta \mathrm{Ala}(N$-Boc)-OMe and 7-azaindole to prepare this compound; in this case the crude product was crystalline and did not require further purification $(92 \%), \mathrm{mp} 91.5-92.0{ }^{\circ} \mathrm{C}$ (from diethyl ether- $n$-hexane) (Found: C, 65.2; H, 6.1; N, 9.9. Calc. for $\mathrm{C}_{23} \mathrm{H}_{25} \mathrm{~N}_{3} \mathrm{O}_{5}$ : C, 65.2; H, 5.95; N, 9.9\%); $\delta_{\mathrm{H}} 0.98(9 \mathrm{H}, \mathrm{s}, \mathrm{Boc})$, $3.84(3 \mathrm{H}, \mathrm{s}, \mathrm{OMe}), 5.11\left(2 \mathrm{H}, \mathrm{m}, \beta \mathrm{CH}_{2}\right), 5.82(1 \mathrm{H}, \mathrm{dd}, J 5.7$ and 9.6, $\alpha \mathrm{CH}), 6.43(1 \mathrm{H}, \mathrm{d}, J 3.6,3-\mathrm{H}$ ind. $), 7.04(1 \mathrm{H}, \mathrm{dd}, J 4.8$ and 7.9, 5- $\mathrm{H}$ ind. $), 7.21-7.40(6 \mathrm{H}, \mathrm{m}, \mathrm{Bz}+2 \mathrm{H}$ ind. $), 7.85(1 \mathrm{H}$, dd, $J 1.5$ and $7.9,4-\mathrm{H}$ ind.), $8.31(1 \mathrm{H}$, dd, $J 1.5$ and $4.8,6-\mathrm{H}$ ind.); $\delta_{\mathrm{C}} 27.05,44.09,52.63,57.50,83.67,100.01,115.97$, $120.66,127.47,127.78,128.64,128.70,131.07,136.80,143.00$, $147.61,152.18,169.16,172.87$.

Synthesis of $\mathbf{3 b}$. The above general method was used with $\mathrm{Bz}\left(\mathrm{NO}_{2}\right)-\Delta \mathrm{Ala}(N$-Boc)-OMe and 1,2,4-triazole to prepare this compound as an oil which failed to crystallise $(67 \%) ; \delta_{\mathrm{H}} 1.12$ ( $9 \mathrm{H}, \mathrm{s}, \mathrm{Boc}), 3.83(3 \mathrm{H}, \mathrm{s}, \mathrm{OMe}), 4.98\left(2 \mathrm{H}, \mathrm{m}, \beta_{\mathrm{CH}_{2}}\right), 5.66$ $(1 \mathrm{H}, \mathrm{dd}, J 6.6$ and $8.3, \alpha \mathrm{CH}), 7.57$ [2H, d, $\left.J 8.7, \mathrm{Bz}\left(\mathrm{NO}_{2}\right)\right]$, 7.93 (1 H, s, 3- or 5-H triaz.), $8.16(1 \mathrm{H}, \mathrm{s}, 5-$ or 3-H triaz.), $8.25\left[2 \mathrm{H}, \mathrm{d}, J 8.7, \mathrm{Bz}\left(\mathrm{NO}_{2}\right)\right] ; \delta_{\mathrm{C}} 27.24,48.13,53.10,57.13$, $85.64,123.34,128.21,142.29,144.15,149.03,151.33,152.46$, $167.88,170.73$.

Synthesis of 3j. The above general method was used with $\mathrm{Bz}\left(\mathrm{NO}_{2}\right)-\Delta \mathrm{Ala}(N$-Boc)-OMe and 7-azaindole to prepare this compound as an oil which failed to crystallise $(54 \%) ; \delta_{\mathrm{H}} 1.07$ $(9 \mathrm{H}, \mathrm{s}, \mathrm{Boc}), 3.86(3 \mathrm{H}, \mathrm{s}, \mathrm{OMe}), 5.04(1 \mathrm{H}, \mathrm{dd}, J 5.1$ and 14.6, $\left.\beta \mathrm{CH}_{2}\right), 5.17\left(1 \mathrm{H}, \mathrm{dd}, J 10.5\right.$ and $\left.14.6, \beta \mathrm{CH}_{2}\right), 5.85(1 \mathrm{H}, \mathrm{dd}$, $J 5.1$ and $10.5, \alpha \mathrm{CH}), 6.47(1 \mathrm{H}, \mathrm{d}, J 3.6,3-\mathrm{H}$ ind.), 7.06-7.21 $\left[4 \mathrm{H}, \mathrm{m}, \mathrm{Bz}\left(\mathrm{NO}_{2}\right)+2-+5-\mathrm{H}\right.$ ind.], $7.88(1 \mathrm{H}, \mathrm{dd}, J 1.5$ and 7.8 , $4-\mathrm{H}$ ind.), 8.10 [2 H, d, $J$ 9.0, $\left.\mathrm{Bz}\left(\mathrm{NO}_{2}\right)\right], 8.30(1 \mathrm{H}, \mathrm{dd}, J 1.5$ and $4.5,6-\mathrm{H}$ ind.); $\delta_{\mathrm{C}} 27.17,43.78,52.79,57.38,84.89,100.38$, $116.19,120.56,123.04,127.87,128.34,128.94,142.50,143.09$, $147.66,148.66,151.26,168.68,170.74$.

Synthesis of 4a. The above general method was used with $\mathrm{Z}-\Delta \mathrm{Ala}(N$-Boc)-OMe and pyrazole to prepare this compound as an oil which failed to crystallise (48\%) (Found: C, 59.5; H, 6.25; N, 10.4. Calc. for $\mathrm{C}_{20} \mathrm{H}_{25} \mathrm{~N}_{3} \mathrm{O}_{6}: \mathrm{C}, 59.5 ; \mathrm{H}, 6.1 ; \mathrm{N}, 10.3 \%$ ); $\delta_{\mathrm{H}} 1.41(9 \mathrm{H}, \mathrm{s}, \mathrm{Boc}), 3.70(3 \mathrm{H}, \mathrm{s}, \mathrm{OMe}), 4.64(1 \mathrm{H}, \mathrm{dd}, J 9.3$ and $\left.14.4, \beta \mathrm{CH}_{2}\right), 4.89\left(1 \mathrm{H}, \mathrm{dd}, J 4.5\right.$ and $\left.14.4, \beta \mathrm{CH}_{2}\right), 5.16(2 \mathrm{H}, \mathrm{s}$, $\left.\mathrm{CH}_{2} \mathrm{Z}\right), 5.48(1 \mathrm{H}, \mathrm{dd}, J 4.5$ and $9.3, \alpha \mathrm{CH}), 6.18(1 \mathrm{H}, \mathrm{t}, J 2.1$, 4-H pyr.), 7.27 (1 H, d, J 2.1, 3- or 5-H pyr.), 7.37 (5 H, m, ArH $\mathrm{Z}), 7.47\left(1 \mathrm{H}, \mathrm{d}, J 2.1\right.$, 5- or $3-\mathrm{H}$ pyr.); $\delta_{\mathrm{C}} 27.69,51.14,52.43$, $58.59,68.85,84.08,105.60,128.32,128.36,128.40,130.07$, $134.89,139.99,150.77,152.96,168.65$.

Synthesis of $\mathbf{4 b}$. The above general method was used with $\mathrm{Z}-\Delta \mathrm{Ala}(N$-Boc)-OMe and 1,2,4-triazole to prepare this compound as an oil which failed to crystallise $(98 \%) ; \delta_{\mathrm{H}} 1.30(9 \mathrm{H}, \mathrm{s}$, Boc), 3.60 (3 H, s, OMe), $4.59\left(1 \mathrm{H}, \mathrm{dd}, J 8.7\right.$ and $\left.14.4, \beta \mathrm{CH}_{2}\right)$, $4.81\left(1 \mathrm{H}, \mathrm{dd}, J 5.1\right.$ and $\left.14.4, \beta_{C_{2}}\right), 5.08\left(2 \mathrm{H}, \mathrm{s}, \mathrm{CH}_{2} \mathrm{Z}\right), 5.38$ 
(1 H, dd, $J 8.7$ and 5.1, $\alpha \mathrm{CH}), 7.27(5 \mathrm{H}, \mathrm{m}, \operatorname{ArH~Z}), 7.77(1 \mathrm{H}$, s, 3- or 5-H triaz.), $7.89\left(1 \mathrm{H}, \mathrm{s}, 5-\right.$ or $3-\mathrm{H}$ triaz.); $\delta_{\mathrm{C}} 27.73,51.18$, $52.47,58.63,68.89,84.13,105.64,128.36,128.44,130.11$, $134.92,140.05,150.79,152.99,168.69$.

Synthesis of $\mathbf{4 j}$. The above general method was used with $\mathrm{Z}-\Delta \mathrm{Ala}(N$-Boc)-OMe and 7-azaindole to prepare this compound as an oil which failed to crystallise $(99 \%) ; \delta_{\mathrm{H}} 1.22(9 \mathrm{H}, \mathrm{s}$, Boc), 3.72 (3 H, s, OMe), $4.76\left(1 \mathrm{H}, \mathrm{dd}, J 9.6\right.$ and $\left.14.6, \beta \mathrm{CH}_{2}\right)$, 4.96-5.11 $\left(3 \mathrm{H}, \mathrm{m}, \beta_{\mathrm{CH}_{2}}+\mathrm{CH}_{2} \mathrm{Z}\right), 5.54(1 \mathrm{H}, \mathrm{dd}, J 4.5$ and 9.6, $\alpha \mathrm{CH}), 6.39$ (1 H, d, $J 3.6,3-\mathrm{H}$ ind.), $7.01(1 \mathrm{H}, \mathrm{d}, J 3.6$, 2-H ind.), 7.04 ( $1 \mathrm{H}$, dd, $J 4.7$ and $7.8,5-\mathrm{H}$ ind.), 7.23-7.35 ( $5 \mathrm{H}, \mathrm{m}, \mathrm{ArH} \mathrm{Z}), 7.86(1 \mathrm{H}$, dd, $J 1.8$ and $7.8,4-\mathrm{H}$ ind.), 8.26 $\left(1 \mathrm{H}\right.$, dd, $J 1.8$ and $4.7,6-\mathrm{H}$ ind.); $\delta_{\mathrm{C}} 27.45,43.96,52.38$, $58.17,68.65,83.78,99.97,115.91,120.55,128.23,128.29$, $128.38,128.50,128.63,134.91,142.86,147.45,150.49,152.91$, 168.87 .

Synthesis of 5a. The above general method was used with $\mathrm{Z}\left(\mathrm{NO}_{2}\right)-\Delta \mathrm{Ala}(N$-Boc)-OMe and pyrazole to prepare this compound as a crystalline material $(37 \%)$, mp $83-84{ }^{\circ} \mathrm{C}$ (from diethyl ether- $n$-hexane) (Found: C, $53.5 ; \mathrm{H}, 5.35 ; \mathrm{N}, 12.8$. Calc. for $\left.\mathrm{C}_{20} \mathrm{H}_{24} \mathrm{~N}_{4} \mathrm{O}_{8}: \mathrm{C}, 53.6 ; \mathrm{H}, 5.4 ; \mathrm{N}, 12.5 \%\right) ; \delta_{\mathrm{H}} 1.45(9 \mathrm{H}, \mathrm{s}, \mathrm{Boc})$, $3.75(3 \mathrm{H}, \mathrm{s}, \mathrm{OMe}), 4.67\left(1 \mathrm{H}, \mathrm{dd}, J 9.6\right.$ and $\left.14.4, \beta_{\mathrm{CH}}\right), 4.90$ $\left(1 \mathrm{H}, \mathrm{dd}, J 4.8\right.$ and $\left.14.4, \beta \mathrm{CH}_{2}\right), 5.26\left[2 \mathrm{H}, \mathrm{s}, \mathrm{CH}_{2} \mathrm{Z}\left(\mathrm{NO}_{2}\right)\right], 5.51$ $(1 \mathrm{H}, \mathrm{dd}, J 4.8$ and 9.6, $\alpha \mathrm{CH}), 6.20(1 \mathrm{H}, \mathrm{t}, J 2.1,4-\mathrm{H}$ pyr. $), 7.32$ (1 H, d, $J 2.4,3-$ or 5-H pyr.), 7.53 [2 H, d, $J 9.0$, $\operatorname{ArH~Z}\left(\mathrm{NO}_{2}\right)$ ], $7.63(1 \mathrm{H}, \mathrm{d}, J 1.8,5-$ or $3-\mathrm{H}$ pyr.), 8.23 [2 H, d, $J$ 9.0, ArH $\left.\mathrm{Z}\left(\mathrm{NO}_{2}\right)\right] ; \delta_{\mathrm{C}} 27.78,51.07,52.65,58.86,67.26,84.61,105.78$, $123.69,123.95,128.16,130.12,140.12,142.33,150.29,153.09$, 168.50 .

Synthesis of $\mathbf{5 b}$. The above general method was used with $\mathrm{Z}\left(\mathrm{NO}_{2}\right)-\Delta \mathrm{Ala}(N$-Boc)-OMe and 1,2,4-triazole to prepare this compound as a crystalline material $(98 \%), \mathrm{mp} 124-125^{\circ} \mathrm{C}$ (from ethyl acetate-diethyl ether) (Found: C, 50.55; H, 5.1; N, 15.6. Calc. for $\left.\mathrm{C}_{19} \mathrm{H}_{23} \mathrm{~N}_{5} \mathrm{O}_{8}: \mathrm{C}, 50.8 ; \mathrm{H}, 5.2 ; \mathrm{N}, 15.6 \%\right) ; \delta_{\mathrm{H}} 1.45$ (9 H, s, Boc), 3.77 ( $3 \mathrm{H}, \mathrm{s}, \mathrm{OMe}), 4.73(1 \mathrm{H}, \mathrm{dd}, J 8.8$ and 14.4 , $\left.\beta \mathrm{CH}_{2}\right), 4.94\left(1 \mathrm{H}, \mathrm{dd}, J 5.4\right.$ and $\left.14.4, \beta \mathrm{CH}_{2}\right), 5.29\left[2 \mathrm{H}, \mathrm{s}, \mathrm{CH}_{2}\right.$ $\mathrm{Z}\left(\mathrm{NO}_{2}\right)$ ], $5.49(1 \mathrm{H}, \mathrm{dd}, J 5.4$ and $8.8, \alpha \mathrm{CH}), 7.55[2 \mathrm{H}, \mathrm{d}, J 8.7$, $\operatorname{ArH~Z}\left(\mathrm{NO}_{2}\right)$ ], $7.90(1 \mathrm{H}, \mathrm{s}, 3-$ or 5-H triaz. $), 8.06(1 \mathrm{H}, \mathrm{s}, 5-$ or $3-\mathrm{H}$ triaz.), 8.26 [2 H, d, $J$ 8.7, $\left.\mathrm{ArH} \mathrm{Z}\left(\mathrm{NO}_{2}\right)\right]$; $\delta_{\mathrm{C}} 27.77,48.69$, $52.86,58.03,67.57,85.10,123.78,128.26,142.03,144.09$, $147.83,150.33,152.34,153.21,168.12$.

Synthesis of 5g. The above general method was used with $\mathrm{Z}\left(\mathrm{NO}_{2}\right)-\Delta \mathrm{Ala}(N$-Boc)-OMe and indole but on a 5 -mmolar scale to prepare this compound as an oil which failed to crystallise $(46 \%) ; \delta_{\mathrm{H}} 1.32(9 \mathrm{H}, \mathrm{s}, \mathrm{Boc}), 3.79(3 \mathrm{H}, \mathrm{s}, \mathrm{OMe}), 4.74(1 \mathrm{H}, \mathrm{dd}$, $J 9.6$ and $\left.15.0, \beta \mathrm{CH}_{2}\right), 4.89\left(1 \mathrm{H}, \mathrm{dd}, J 4.8\right.$ and $\left.15.0, \beta \mathrm{CH}_{2}\right), 5.00$ [2 $\mathrm{H}, 2 \mathrm{~d}, J 13.5, \mathrm{CH}_{2} \mathrm{Z}\left(\mathrm{NO}_{2}\right)$ ], $5.38(1 \mathrm{H}, \mathrm{dd}, J 4.8$ and 9.6, $\alpha \mathrm{CH}), 6.47(1 \mathrm{H}, \mathrm{dd}, J 0.9$ and $3.2,3-\mathrm{H}$ ind.), $7.00(1 \mathrm{H}, \mathrm{d}, J 3.2$, 2- $\mathrm{H}$ ind.), 7.08-7.31 [5 H, m, 5-, 6- and 7-H ind. $+\mathrm{ArH}$ $\left.\mathrm{Z}\left(\mathrm{NO}_{2}\right)\right], 7.60(1 \mathrm{H}, \mathrm{d}, J 8.1,4-\mathrm{H}$ ind.), $8.16[2 \mathrm{H}, \mathrm{d}, J 9.0, \mathrm{ArH}$ $\left.\mathrm{Z}\left(\mathrm{NO}_{2}\right)\right] ; \delta_{\mathrm{C}} 27.58,45.53,52.66,58.69,67.18,84.61,102.20$, $108.90,119.70,121.07,121.84,123.62,128.13,128.58,128.64$, $136.07,142.04,147.93,150.33,153.00,168.62$.

Synthesis of $5 \mathbf{j}$. The above general method was used with $\mathrm{Z}\left(\mathrm{NO}_{2}\right)-\Delta \mathrm{Ala}(N$-Boc)-OMe and 7-azaindole to prepare this compound as an oil which failed to crystallise $(87 \%) ; \delta_{\mathrm{H}} 1.30$ ( $9 \mathrm{H}, \mathrm{s}, \mathrm{Boc}), 3.78$ ( $3 \mathrm{H}, \mathrm{s}, \mathrm{OMe}), 4.83(1 \mathrm{H}, \mathrm{dd}, J 9.9$ and 14.6, $\left.\beta_{\mathrm{CH}_{2}}\right), 4.87-5.15\left[3 \mathrm{H}, \mathrm{m}, \beta_{\mathrm{CH}}+\mathrm{CH}_{2} \mathrm{Z}\left(\mathrm{NO}_{2}\right)\right], 5.56(1 \mathrm{H}, \mathrm{dd}$, $J 9.9$ and $4.5, \alpha \mathrm{CH}), 6.40(1 \mathrm{H}, \mathrm{d}, J 3.3,3-\mathrm{H}$ ind.), 7.01-7.08 $\left(2 \mathrm{H}, \mathrm{m}, 2-+5-\mathrm{H}\right.$ ind.), $7.34\left[2 \mathrm{H}, \mathrm{d}, J 8.4, \mathrm{ArH} \mathrm{Z}\left(\mathrm{NO}_{2}\right)\right], 7.85$ $(1 \mathrm{H}, \mathrm{dd}, J 1.5$ and $7.5,4-\mathrm{H}$ ind.), 8.17 [2 H, d, $J$ 8.4, ArH $\left.\mathrm{Z}\left(\mathrm{NO}_{2}\right)\right], 8.24(1 \mathrm{H}$, dd, $J 1.5$ and $4.8,6-\mathrm{H}$ ind. $) ; \delta_{\mathrm{C}} 27.57,43.96$, $52.58,58.52,67.02,84.33,100.15,116.02,120.52,123.64$, $128.00,128.42,128.72,128.89,142.31,142.94,147.53,150.12$, $153.03,168.72$.
Deprotection of $\beta$-substituted $N$-acyl- $N$-(tert-butyloxycarbonyl)alanine methyl esters

General method of acidolysis. To the fully protected amino acid $(1 \mathrm{mmol})$ were added $3 \mathrm{~cm}^{3}$ of TFA at room temperature and the solution was left for $1 \mathrm{~h}$. Excess of TFA was removed by evaporation at reduced pressure to give the required Boc-free amino acid.

General method of saponification. To the fully protected amino acid $(1 \mathrm{mmol})$ in 1,4-dioxane $\left(5 \mathrm{~cm}^{3}\right)$ were added $3 \mathrm{~cm}^{3}$ of $\mathrm{NaOH}\left(1 \mathrm{~mol} \mathrm{dm}^{-3}\right)$. The solution was stirred at room temperature for $2 \mathrm{~h}$, acidified to $\mathrm{pH} 2-3$ with $\mathrm{KHSO}_{4}\left(1 \mathrm{~mol} \mathrm{dm}^{-3}\right)$ and then extracted with ethyl acetate. The organic layer was dried over $\mathrm{MgSO}_{4}$ and evaporated at reduced pressure to give the corresponding acid as required.

Acidolysis of $\mathbf{1 b}$. The above general method of acidolysis was used to give $\mathbf{6 b}$ in $80 \%$ yield, $\mathrm{mp} 115.5-116.5^{\circ} \mathrm{C}$ (from ethyl acetate- $n$-hexane) (Found: $\mathrm{C}, 30.7 ; \mathrm{H}, 3.3 ; \mathrm{N}, 13.8$. Calc. for $\left.\mathrm{C}_{10} \mathrm{H}_{12} \mathrm{~F}_{6} \mathrm{~N}_{4} \mathrm{O}_{6}: \mathrm{C}, 30.2 ; \mathrm{H}, 3.0 ; \mathrm{N}, 14.1 \%\right) ; \delta_{\mathrm{H}} 3.74(3 \mathrm{H}, \mathrm{s}$, OMe), $4.65\left(3 \mathrm{H}, \mathrm{m}, \beta \mathrm{CH}_{2}+\alpha \mathrm{CH}\right), 8.05(1 \mathrm{H}, \mathrm{s}, 3-$ or $5-\mathrm{H}$ triaz.), 8.51 ( $1 \mathrm{H}, \mathrm{s}, 5-$ or $3-\mathrm{H}$ triaz.), 8.65 (3 H, broad signal, s, $\left.\mathrm{NH}_{3}\right) ; \delta_{\mathrm{C}}(\mathrm{DMSO}) 47.72,51.54,53.20,145.46,152.21,167.53$.

Acidolysis of 1j. The above general method of acidolysis was used to give $6 \mathbf{j}$ in $85 \%$ yield, mp $132-133{ }^{\circ} \mathrm{C}$ (from ethyl acetate$n$-hexane) (Found: $\mathrm{C}, 46.7 ; \mathrm{H}, 4.35 ; \mathrm{N}, 12.8$. Calc. for $\mathrm{C}_{13} \mathrm{H}_{14^{-}}$ $\left.\mathrm{F}_{3} \mathrm{~N}_{3} \mathrm{O}_{4}: \mathrm{C}, 46.85 ; \mathrm{H}, 4.2 ; \mathrm{N}, 12.6 \%\right) ; \delta_{\mathrm{H}}(\mathrm{DMSO}) 3.70(3 \mathrm{H}, \mathrm{s}$, OMe), $4.86\left(3 \mathrm{H}, \mathrm{m}, \mathrm{BCH}_{2}+\alpha \mathrm{CH}\right), 6.52(1 \mathrm{H}, \mathrm{d}, J 3.6,3-\mathrm{H}$ ind.), 7.17 ( $2 \mathrm{H}, \mathrm{m}, 5-+2-\mathrm{H}$ ind.), $8.01(1 \mathrm{H}, \mathrm{dd}, J 1.5$ and 8.1 , $4-\mathrm{H}$ ind.), 8.27 ( $1 \mathrm{H}$, dd, $J 1.5$ and $4.8,6-\mathrm{H}$ ind.); $\delta_{\mathrm{C}}$ (DMSO) $44.08,52.02,52.94,100.39,116.24,120.49,128.99,129.69$, $142.45,147.43,168.15$.

Acidolysis of 1m. The above general method of acidolysis was used to give $6 \mathrm{~m}$ in a $91 \%$ yield, $\mathrm{mp} 186-186.5^{\circ} \mathrm{C}$ (from ethyl acetate- $n$-hexane) (Found: C, 56.4; H, 4.75; N, 7.1. Calc. for $\mathrm{C}_{18} \mathrm{H}_{17} \mathrm{~F}_{3} \mathrm{~N}_{2} \mathrm{O}_{4}$ : C, 56.55; H, 4.5; N, 7.3\%); $\delta_{\mathrm{H}}$ (DMSO) 3.32 $(3 \mathrm{H}, \mathrm{s}, \mathrm{OMe}), 4.45\left(2 \mathrm{H}, \mathrm{t}, J 7.2, \beta \mathrm{CH}_{2}\right), 4.81(1 \mathrm{H}, \mathrm{d}, J$ 7.2, $\alpha \mathrm{CH}$ ), 7.25 (2 H, t, J 8.1, 1- and 8-H carb.), 7.49 (4 H, m, 2-, 3-, 6- and 7-H carb.), 8.18 ( $2 \mathrm{H}, \mathrm{d}, J$ 7.5, 4- and 5-H carb.), 8.71 (3 H, broad signal, $\mathrm{NH}_{3}$ ); $\delta_{\mathrm{C}}(\mathrm{DMSO}) 42.51,50.44,52.69$, $108.92,119.57,120.45,122.60,125.98,139.97,168.64$.

Aminolysis of 2b. DEAEA-induced aminolysis of $\mathbf{2 b}$ was carried out according to the procedure described by Ragnarsson and co-workers ${ }^{13}$ to give $7 \mathbf{b}(78 \%), \mathrm{mp} 76-78^{\circ} \mathrm{C}$ (from diethyl ether-n-hexane) (Found: $\mathrm{C}, 49.0 ; \mathrm{H}, 6.5 ; \mathrm{N}, 20.8$. Calc. for $\mathrm{C}_{11} \mathrm{H}_{18} \mathrm{~N}_{4} \mathrm{O}_{4}$ : C, 48.9; H, 6.7; N, 20.7\%); $\delta_{\mathrm{H}} 1.44(9 \mathrm{H}, \mathrm{s}, \mathrm{Boc})$, $3.80(3 \mathrm{H}, \mathrm{s}, \mathrm{OMe}), 4.67\left(3 \mathrm{H}\right.$, broad signal, $\left.\alpha \mathrm{CH}+\beta \mathrm{CH}_{2}\right), 5.38$ ( $1 \mathrm{H}$, broad signal, $\mathrm{NH}), 7.94(1 \mathrm{H}, \mathrm{s}, 3-$ or $5-\mathrm{H}$ triaz. $), 8.02$ $\left(1 \mathrm{H}, \mathrm{s}, 5-\right.$ or $3-\mathrm{H}$ triaz.); $\delta_{\mathrm{C}} 28.17,50.07,53.10,53.41,80.66$, $144.16,152.46,155.03,169.61$.

$\mathbf{A l}(\mathbf{H g})$-Mediated cleavage of $\mathbf{5 b}$. Finely cut aluminium foil (10 mmol) was stirred with a few drops of mercury for $30 \mathrm{~min}$ under a stream of nitrogen; a solution of $\mathbf{5 b}(0.5 \mathrm{mmol})$ in THF $\left(5 \mathrm{~cm}^{3}\right)$ with $1 \%$ water was then added. After $2 \mathrm{~h}$, when most of the Al had dissolved and TLC indicated only minor amounts of starting material, more $\mathrm{Al}(\mathrm{Hg})(5 \mathrm{mmol})$ was added and left to react for $2 \mathrm{~h}$. The greyish solid material was then filtered off by suction and rinsed thoroughly with ethyl acetate. Evaporation of the filtrate gave an oil which was chromatographed by column chromatography through silica with ethyl acetate-diethyl ether $1: 1$ as eluent to give $7 \mathbf{b}(86 \%)$.

Saponification of 1a. The above general method of saponification was used to give 8a (91\%), mp $149-150{ }^{\circ} \mathrm{C}$ (from ethyl acetate- $n$-hexane) (Found: C, 54.2; H, 7.1; N, 11.9. Calc. for 
$\left.\mathrm{C}_{16} \mathrm{H}_{25} \mathrm{~N}_{3} \mathrm{O}_{6}: \mathrm{C}, 54.1 ; \mathrm{H}, 7.1 ; \mathrm{N}, 11.8 \%\right) ; \delta_{\mathrm{H}}$ (DMSO) $1.48(18 \mathrm{H}$, s, Boc), $4.67\left(1 \mathrm{H}, \mathrm{dd}, J 8.7\right.$ and $\left.14.5, \beta \mathrm{CH}_{2}\right), 5.06(1 \mathrm{H}, \mathrm{dd}, J 4.8$ and $\left.14.5, \beta \mathrm{CH}_{2}\right), 5.47(1 \mathrm{H}, \mathrm{dd}, J 4.8$ and $8.7, \alpha \mathrm{CH}), 6.28(1 \mathrm{H}$, t, $J$ 2.4, 4-H pyr.), 7.39 (1 H, d, $J 2.4,3$ - or 5-H pyr.), $7.58(1 \mathrm{H}$, d, $J 1.8,5$ - or $3-\mathrm{H}$ pyr.); $\delta_{\mathrm{C}}$ (DMSO) $27.48,50.74,58.21,82.40$, $105.22,130.52,139.01,150.94,169.86$.

Saponification of $\mathbf{1 b}$. The above general method of saponification was used to give $\mathbf{8 b}(86 \%)$, mp $129-129.5^{\circ} \mathrm{C}$ (from ethyl acetate- $n$-hexane) (Found: $\mathrm{C}, 50.7 ; \mathrm{H}, 7.0 ; \mathrm{N}, 15.2$. Calc. for $\mathrm{C}_{15} \mathrm{H}_{24} \mathrm{~N}_{4} \mathrm{O}_{6}$ : C, 50.55; H, 6.8; N, 15.7\%); $\delta_{\mathrm{H}}$ (DMSO) 1.47 (18 $\mathrm{H}, \mathrm{s}, \mathrm{Boc}), 4.70\left(1 \mathrm{H}, \mathrm{dd}, \beta \mathrm{CH}_{2}\right), 4.96\left(1 \mathrm{H}, \mathrm{dd}, \beta \mathrm{CH}_{2}\right), 5.44(1$ $\mathrm{H}, \mathrm{dd}, \alpha \mathrm{CH}), 7.97(1 \mathrm{H}, \mathrm{s}, 3-$ or 5-H triaz.), 8.23 (1 H, s, 5- or $3-\mathrm{H}$ triaz.); $\delta_{\mathrm{C}}$ (DMSO) 27.43, 48.23, 57.73, 82.65, 144.78, $150.90,151.47,169.51$

Saponification of $\mathbf{1 h}$. The above general method of saponification was used to give $\mathbf{8 h}(89 \%), \mathrm{mp} 155-156^{\circ} \mathrm{C}$ (from ethyl acetate $-n$-hexane) (Found: $\mathrm{C}, 61.05 ; \mathrm{H}, 6.6 ; \mathrm{N}, 6.5$. Calc. for $\left.\mathrm{C}_{22} \mathrm{H}_{28} \mathrm{~N}_{2} \mathrm{O}_{7}: \mathrm{C}, 61.1 ; \mathrm{H}, 6.5 ; \mathrm{N}, 6.5 \%\right) ; \delta_{\mathrm{H}}$ (DMSO) $1.32(18 \mathrm{H}$, s, Boc), $4.81\left(1 \mathrm{H}, \mathrm{dd}, J 9.3\right.$ and $\left.14.7, \beta \mathrm{CH}_{2}\right), 4.93(1 \mathrm{H}, \mathrm{dd}, J 4.8$ and $\left.14.7, \beta \mathrm{CH}_{2}\right), 5.44(1 \mathrm{H}, \mathrm{dd}, J 4.8$ and $9.3, \alpha \mathrm{CH}), 7.27-7.44$ (3 H, complex signal, 5-, 6- and 7- $\mathrm{H}$ ind.), 7.75 (1 H, s, 2- $\mathrm{H}$ ind.), 8.30 ( $1 \mathrm{H}, \mathrm{d}, J$ 5.4, 4-H ind.), $9.99(1 \mathrm{H}, \mathrm{s}, \mathrm{CHO}) ; \delta_{\mathrm{c}}$ (DMSO) 27.17, 46.03, 57.84, 82.65, 110.70, 117.76, 120.99, $122.45,123.55,124.64,137.44,141.27,151.07,169.61,184.64$.

Saponification of $\mathbf{1 j}$. The above general method of saponification was used to give $8 \mathbf{j}(94 \%), \mathrm{mp} 156-156.5^{\circ} \mathrm{C}$ (from ethyl acetate- $n$-hexane) (Found: $\mathrm{C}, 59.3 ; \mathrm{H}, 6.8 ; \mathrm{N}, 10.0$. Calc. for $\left.\mathrm{C}_{20} \mathrm{H}_{27} \mathrm{~N}_{3} \mathrm{O}_{6}: \mathrm{C}, 59.25 ; \mathrm{H}, 6.7 ; \mathrm{N}, 10.4 \%\right) ; \delta_{\mathrm{H}}$ (DMSO) 1.30 (18 $\mathrm{H}, \mathrm{s}, \mathrm{Boc}), 4.67(1 \mathrm{H}, \mathrm{dd}, J 10.2$ and $14.7, \alpha \mathrm{CH}), 5.45(2 \mathrm{H}, \mathrm{m}$, $\left.\beta \mathrm{CH}_{2}\right), 6.51(1 \mathrm{H}, \mathrm{d}, J 3.3,3-\mathrm{H}$ ind.), $7.13(1 \mathrm{H}, \mathrm{d}, J 3.3,2-\mathrm{H}$ ind.), 7.21 ( $1 \mathrm{H}$, dd, $J 4.8$ and 7.8, 5- $\mathrm{H}$ ind.), $8.04(1 \mathrm{H}, \mathrm{d}, J 7.8$, 4- $\mathrm{H}$ ind.), 8.42 (1 H, d, $J$ 4.8, 6- $\mathrm{H}$ ind.); $\delta_{\mathrm{C}}$ (DMSO) 27.74 , $44.12,58.33,82.65,99.91,116.17,120.64,128.85,129.79$, $142.79,147.76,151.19,170.51$.

\section{Coupling reactions of $\beta$-substituted alanine derivatives}

Synthesis of Boc-DL-Ala[ $N$-Boc- $\beta$-(pyrazol-1-yl)]-L-Phe-OEt 9a. Compound 8a was treated on a 1-mmolar scale with H-Phe$\mathrm{OEt} \cdot \mathrm{HCl}$ in ethyl acetate by using the standard DCC-1hydroxybenzotriazole(HOBt) procedure to give Boc-Ala[ $N$-Boc- $\beta$-(pyrazol-1-yl)]-L-Phe-OEt as a pure oil that solidified on storage $(80 \%)$ (Found: $\mathrm{C}, 61.0 ; \mathrm{H}, 7.3 ; \mathrm{N}, 10.45$. Calc. for $\mathrm{C}_{27} \mathrm{H}_{38} \mathrm{~N}_{4} \mathrm{O}_{7}$ : C, 61.1; H, 7.2; N, 10.6\%).

Synthesis of Boc-DL-Ala[ $N$-Boc- $\beta$-(7-azaindol-1-yl)]-L-PheOEt 9j. Compound $\mathbf{8 j}$ was treated on a $1-$ mmolar scale as above to give Boc-Ala[ $N$-Boc- $\beta$-(7-azaindol-1-yl)]-L-Phe-OEt as a pure oil that solidified on storage $(86 \%)$ (Found: $\mathrm{C}, 64.05 ; \mathrm{H}$, 7.1; N, 9.7. Calc. for $\mathrm{C}_{31} \mathrm{H}_{40} \mathrm{~N}_{4} \mathrm{O}_{7}$ : C, 64.1; H, 6.9; N, 9.65\%).

\section{Michael addition to dehydroalanine dipeptides}

General method. To a solution of $0.25 \mathrm{mmol}$ of the fully protected dehydro dipeptide in acetonitrile, viz. Tos-Gly $(N$ Boc)- $\Delta \mathrm{Ala}(N$-Boc)-OMe 10 or Boc-Ala $(N$-Boc)- $\Delta \mathrm{Ala}(N$-Boc)OMe $11\left(2.5 \mathrm{~cm}^{3}\right)$ was added $\mathrm{K}_{2} \mathrm{CO}_{3}$ (6 equiv.), followed by 1 equiv. of pyrazole with rapid stirring at room temperature. The reaction was monitored by TLC and when no starting material was detected the solution was filtered, and evaporated at reduced pressure to give the corresponding addition product.

Synthesis of Tos-Gly $(N$-Boc)-Ala[ $N$-Boc- $\beta$-(pyrazol-1-yl)]OMe. The above general method with pyrazole gave TosGly( $N$-Boc)-Ala[ $N$-Boc- $\beta$-(pyrazol-1-yl)]-OMe 10a in $76 \%$ yield, $\mathrm{mp} 129.5-131^{\circ} \mathrm{C}$ (from diethyl ether- $n$-hexane) (Found: C, 53.6; H, 6.25; N, 9.3; S, 5.7. Calc. for $\mathrm{C}_{26} \mathrm{H}_{36} \mathrm{~N}_{4} \mathrm{O}_{9} \mathrm{~S}$ : C, 53.8; $\mathrm{H}, 6.25 ; \mathrm{N}, 9.65 ; \mathrm{S}, 5.5 \%) ; \delta_{\mathrm{H}} 1.33(9 \mathrm{H}, \mathrm{s}, \mathrm{Boc}), 1.44(9 \mathrm{H}, \mathrm{s}$,
Boc), 2.45 (3 H, s, $\mathrm{CH}_{3}$ Tos), 3.76 (3 H, s, OMe), 4.59 (1 H, dd, $J 9.6$ and $\left.14.4, \beta_{C_{2}} \mathrm{Ala}\right), 4.94\left(1 \mathrm{H}, \mathrm{dd}, J 4.5\right.$ and $14.4, \beta \mathrm{CH}_{2}$ Ala), $5.10\left(2 \mathrm{H}, \mathrm{d}, J 6.9, \mathrm{CH}_{2} \mathrm{Gly}\right), 5.50(1 \mathrm{H}, \mathrm{dd}, J 4.5$ and 9.6, aCH Ala), 6.23 (1 H, t, J 2.1, 4-H pyr.), 7.30 (2 H, d, $J$ 8.1, ArH Tos), $7.45(1 \mathrm{H}, \mathrm{d}, J 2.1,3-$ or $5-\mathrm{H}$ pyr.), $7.53(1 \mathrm{H}, \mathrm{d}, J 1.5,5$ - or 3-H pyr.), 7.87 (2 H, d, $J$ 8.1, ArH Tos); $\delta_{\mathrm{C}} 21.62,27.71,27.76$, 50.27, 51.54, 52.49, 57.70, 84.63, 85.43, 105.86, 128.69, 128.98, $130.45,136.82,140.14,144.22,150.54,151.14,168.30,170.63$.

Synthesis of Tos-Gly( $N$-Boc)-Ala[ $N$-Boc- $\beta-(1,2,4-t r i a z o l-1-$ yl)]-OMe. The above general method with 1,2,4-triazole gave Tos-Gly $(N$-Boc)-Ala[ $N$-Boc- $\beta$-(1,2,4-triazol-1-yl)]-OMe 10b in $99 \%$ yield, mp $130.5-132{ }^{\circ} \mathrm{C}$ (from diethyl ether- $n$-hexane) (Found: C, 51.8; H, 6.1; N, 11.85; S, 5.6. Calc. for $\mathrm{C}_{25} \mathrm{H}_{35} \mathrm{~N}_{5} \mathrm{O}_{9} \mathrm{~S}$ : C, 51.6; H, 6.1; N, 12.0; S, 5.5\%); $\delta_{\mathrm{H}} 1.33(9 \mathrm{H}, \mathrm{s}, \mathrm{Boc}), 1.45$ (9 H, s, Boc), 2.46 (3 H, s, $\mathrm{CH}_{3}$ Tos), 3.78 (3 H, s, OMe), 4.62 $\left(1 \mathrm{H}\right.$, dd, $J 9.0$ and $14.4, \beta \mathrm{CH}_{2}$ Ala), $4.94(1 \mathrm{H}, \mathrm{dd}, J 4.5$ and 14.4, $\left.\beta_{C_{2}} \mathrm{Ala}\right), 5.08$ ( $\left.2 \mathrm{H}, \mathrm{s}, \mathrm{CH}_{2} \mathrm{Gly}\right), 5.46(1 \mathrm{H}, \mathrm{dd}, J 4.5$ and 9.0, $\alpha \mathrm{CH}$ Ala), 7.32 (2 H, d, $J$ 8.1, ArH Tos), 7.87 ( $2 \mathrm{H}, \mathrm{d}, J 8.1$, ArH Tos), 7.95 (1 H, s, 3- or 5-H triaz.), 8.23 (1 H, s, 5- or 3-H triaz.); $\delta_{\mathrm{C}} 21.61,27.70,27.75,47.84,51.53,52.72,57.01,84.89$, $85.95,128.55,129.05,136.73,144.34,144.43,150.56,150.94$, $152.36,167.89,170.85$.

Synthesis of Tos-Gly $(N$-Boc)-Ala[ $N$-Boc- $\beta$-(imidazol-1-yl)]OMe. The above general method with imidazole gave TosGly $(N$-Boc)-Ala[ $N$-Boc- $\beta$-(imidazol-1-yl)]-OMe 10c in $75 \%$ yield, mp $108-109.5^{\circ} \mathrm{C}$ (from diethyl ether- $n$-hexane) (Found: C, 53.7; H, 6.2; N, 9.4; S, 5.4. Calc. for $\mathrm{C}_{26} \mathrm{H}_{36} \mathrm{~N}_{4} \mathrm{O}_{9} \mathrm{~S}: \mathrm{C}, 53.8 ; \mathrm{H}$, 6.25; N, 9.65; S, 5.5\%); $\delta_{\mathrm{H}} 1.33(9 \mathrm{H}, \mathrm{s}, \mathrm{Boc}), 1.43(9 \mathrm{H}, \mathrm{s}, \mathrm{Boc})$, $2.45\left(3 \mathrm{H}, \mathrm{s}, \mathrm{CH}_{3} \mathrm{Tos}\right), 3.77(3 \mathrm{H}, \mathrm{s}, \mathrm{OMe}), 4.38(1 \mathrm{H}, \mathrm{dd}, J 8.4$ and $\left.14.9, \beta \mathrm{CH}_{2} \mathrm{Ala}\right), 4.70\left(1 \mathrm{H}, \mathrm{dd}, J 4.8\right.$ and $\left.14.9, \beta_{\mathrm{CH}_{2}} \mathrm{Ala}\right)$, $5.11\left(2 \mathrm{H}, \mathrm{d}, J 10.2, \mathrm{CH}_{2} \mathrm{Gly}\right), 5.25(1 \mathrm{H}, \mathrm{dd}, J 4.8$ and $8.4, \alpha \mathrm{CH}$ Ala), 6.96 (1 H, t, $J$ 1.2, 4- or 5-H imid.), 7.04 (1 H, s, 5- or 4-H imid.), 7.33 (2 H, d, $J$ 8.4, ArH Tos), $7.54(1 \mathrm{H}, \mathrm{s}, 2-\mathrm{H}$ imid.), 7.87 (2 H, d, $J$ 8.4, ArH Tos); $\delta_{\mathrm{C}} 21.65,27.69,27.78$, $45.58,51.61,52.71,58.06,84.92,86.18,119.62,128.58$, $129.09,129.74,136.75,137.82,144.39,150.56,150.96,168.10$, 170.76 .

Synthesis of Tos-Gly $(N$-Boc)-Ala[ $N$-Boc- $\beta$-(3-formylindol-1yl)]-OMe. The above general method with 3 -formylindole gave $\operatorname{Tos}-\mathrm{Gly}(N$-Boc)-Ala[ $N$-Boc- $\beta$-(3-formylindol-1-yl)]-OMe $\mathbf{1 0 h}$ in $74 \%$ yield, $\mathrm{mp} 117.0-119.0{ }^{\circ} \mathrm{C}$ (from diethyl ether- $n$-hexane) (Found: C, 58.6; H, 5.9; N, 6.65; S, 4.32. Calc. for $\mathrm{C}_{32} \mathrm{H}_{39} \mathrm{~N}_{3} \mathrm{O}_{10} \mathrm{~S}$ : $\mathrm{C}, 58.4 ; \mathrm{H}, 5.9 ; \mathrm{N}, 6.4 ; \mathrm{S}, 4.86 \%) ; \delta_{\mathrm{H}} 1.06(9 \mathrm{H}, \mathrm{s}, \mathrm{Boc}), 1.35(9 \mathrm{H}$, $\mathrm{s}, \mathrm{Boc}), 2.47$ (3 H, s, $\left.\mathrm{CH}_{3} \mathrm{Tos}\right), 3.80$ (3 H, s, OMe), $4.65(1 \mathrm{H}, \mathrm{dd}$, $J 9.0$ and 15.0, $\beta_{\mathrm{CH}_{2}}$ Ala), $5.00\left(1 \mathrm{H}, \mathrm{dd}, J 4.8\right.$ and $15.0, \beta_{\mathrm{CH}}$ Ala), 5.11 (2 H, d, J 5.7, $\mathrm{CH}_{2}$ Gly), $5.33(1 \mathrm{H}, \mathrm{m}, \alpha \mathrm{CH}$ Ala $)$, 7.29-7.43 (5 H, m, 2-, 5- and 6-H ind. + ArH Tos), 7.87-7.92 $(3 \mathrm{H}, \mathrm{m}, 7-\mathrm{H}$ ind. + ArH Tos), $8.32(1 \mathrm{H}, \mathrm{dd}, J 2.7$ and $6.2,4-\mathrm{H}$ ind.), $9.98(1 \mathrm{H}, \mathrm{s}, \mathrm{CH}) ; \delta_{\mathrm{C}} 21.66,27.16,27.80,45.52,51.75$, $52.78,56.83,85.06,85.97,109.57,118.91,122.65,123.13$, $124.36,125.36,128.46,129.15,136.86,137.10,140.24,144.44$, $150.69,150.82,168.11,171.01,184.99$.

Synthesis of Tos-Gly( $N$-Boc)-Ala[ $N$-Boc- $\beta$-(7-azaindol-1-yl)]OMe. The above general method with 7-azaindole gave TosGly $(N$-Boc)-Ala[ $N$-Boc- $\beta$-(7-azaindol-1-yl)]-OMe 10j in $90 \%$ yield, $\mathrm{mp} 141.5-143{ }^{\circ} \mathrm{C}$ (from diethyl ether- $n$-hexane) (Found: C, 57.0; H, 6.2; N, 8.7; S, 5.2. Calc. for $\mathrm{C}_{30} \mathrm{H}_{38} \mathrm{~N}_{4} \mathrm{O}_{9} \mathrm{~S}: \mathrm{C}, 57.1 ; \mathrm{H}$, $6.1 ; \mathrm{N}, 8.9 ; \mathrm{S}, 5.1 \%) ; \delta_{\mathrm{H}} 1.05(9 \mathrm{H}, \mathrm{s}, \mathrm{Boc}), 1.34(9 \mathrm{H}, \mathrm{s}, \mathrm{Boc})$, $2.45\left(3 \mathrm{H}, \mathrm{s}, \mathrm{CH}_{3} \mathrm{Tos}\right), 3.77(3 \mathrm{H}, \mathrm{s}, \mathrm{OMe}), 4.73(1 \mathrm{H}, \mathrm{dd}, J 9.6$ and 14.6, $\left.\beta_{\mathrm{CH}_{2}} \mathrm{Ala}\right), 5.13$ ( $\left.3 \mathrm{H}, \mathrm{m}, \beta_{\mathrm{CH}} \mathrm{Ala}, \mathrm{CH}_{2} \mathrm{Gly}\right), 5.49$ $(1 \mathrm{H}, \mathrm{dd}, J 3.6$ and 9.6, $\alpha \mathrm{CH}$ Ala), $6.46(1 \mathrm{H}, \mathrm{d}, J 5.1,3-\mathrm{H}$ ind.), 7.07 ( $1 \mathrm{H}, \mathrm{dd}, J 5.1$ and 7.8, 5-H ind.), 7.27 (3 H, m, 2-H ind., ArH Tos), 7.88 (3 H, m, 4-H ind., ArH Tos), 8.35 ( $1 \mathrm{H}$, dd, $J 1.5$ and $5.1,6-\mathrm{H}$ ind.); $\delta_{\mathrm{C}} 21.63,27.20,27.75,51.21,51.75,52.37$, $57.47,84.50,84.80,105.68,128.78,128.91,130.14,136.93$, $140.09,142.94,144.13,151.15,170.70,168.45$. 
Synthesis of Boc-DL-Ala( $N$-Boc)-Ala[ $N$-Boc- $\beta-(1,2,4$-triazol1-yl)]-OMe. The above general method with 1,2,4-triazole gave $\operatorname{Boc}-\mathrm{Ala}(N$-Boc)-Ala[ $N$-Boc- $\beta$-(1,2,4-triazol-1-yl)]-OMe 11b in $98 \%$ yield (from diethyl ether- $n$-hexane) (Found: C, 52.9 ; $\mathrm{H}, 7.1 ; \mathrm{N}, 12.9$. Calc. for $\mathrm{C}_{24} \mathrm{H}_{39} \mathrm{~N}_{5} \mathrm{O}_{9}: \mathrm{C}, 53.2 ; \mathrm{H}, 7.3 ; \mathrm{N}$ $12.9 \%)$.

\section{References}

1 P. M. Dunnill and L. Fowde, Phytochemistry, 1965, 4, 935

2 H. Shinozaki and S. Konishi, Neuropharmacology, 1974, 13, 665.

3 R. H. Evans, A. A. Francis, K. Hunt, M. R. Martin and J. C. Watkins, J. Pharm. Pharmac., 1978, 30, 364.

4 F. Ikegami and I. Murakoshi, Phytochemistry, 1994, 35, 1089.
5 F. Ikegami, Y. Komada, M. Kobori, D. R. Hawkins and I. Murakoshi, Phytochemistry, 1990, 29, 2507.

6 T. Okuda and H. Zahn, Chem. Ber., 1965, 98, 1164.

7 R. Labia and C. Morin, J. Org. Chem., 1986, 51, 249.

8 M. Pérez and R. Pleixats, Tetrahedron, 1995, 51, 8355.

9 M. Barbaste, V. Rolland-Fulcrand, M. L. Roumestant, P. Viallefont and J. Martinez, Tetrahedron Lett., 1998, 39, 6287.

10 P. M. T. Ferreira, H. L. S. Maia and L. S. Monteiro, Tetrahedron Lett., 1998, 39, 9575.

11 P. M. T. Ferreira, H. L. S. Maia, L. S. Monteiro and J. Sacramento, J. Chem. Soc., Perkin Trans. 1, 1999, 3697.

12 P. M. T Ferreira, H. L. S. Maia and L. S. Monteiro, Tetrahedron Lett., 1999, 40, 4099.

13 L. Grehn, K. Gunnarsson and U. Ragnarsson, Acta Chem. Scand., Ser: $B, 1987, \mathbf{4 1}, 18$.

14 B. Nyassé, L. Grehn, H. L. S. Maia, L. S. Monteiro and U. Ragnarsson, J. Org. Chem., 1999, 64, 7135. 